\title{
MODELO PARA DETERMINAR AS QUANTIDADES EM ESTOQUE DE PEÇAS SOBRESSALENTES EM UMA PLANTA DE GERAÇÃO DE ENERGIA
}

\section{MODEL TO DETERMINE THE QUANTITIES OF SPARE PARTS IN STOCK IN A POWER GENERATION PLANT}

\author{
Alberto Magno Teodoro Filho* E-mail: amagnotf@hotmail.com \\ Marcos de Andrade Schroeder*E-mail: meocamp@gmail.com \\ Paulo Sérgio de Arruda Ignácio* E-mail: paulo.ignacio@fca.unicamp.br \\ Gabriel Alves da Costa Lima** E-mail: gabriel.costa.lima@aremas.com.br \\ *Universidade Estadual de Campinas (UNICAMP), Limeira, SP \\ ** AREMAS - Reliability, Risk \& Economics, Campinas, SP
}

\begin{abstract}
Resumo: Neste artigo apresenta-se um modelo para avaliar o tempo total indisponível de uma planta de geração de energia elétrica devido à falta de peças de reposição em função dos níveis de estoque de cada uma destas peças. O modelo considera itens que apareçam uma ou duas vezes no sistema com configuração de confiabilidade do tipo série ou paralelo, com ou sem política de substituição preventiva. Otimização estocástica baseada na metaheurística Scatter Search foi utilizada para definir os níveis de estoque com objetivo de minimizar a média do tempo total indisponível devido à falta de peças e respeitando a restrição estatística sobre o valor total gasto para o horizonte de planejamento. Os resultados indicaram que, por exemplo, se a restrição de custo de $\mathrm{R} \$ 550.000$ para 10 anos for aumentada em 9,1\%, a média do tempo indisponível é reduzida em 55,9\% (de 617 para 272 horas). Portanto, demonstra-se que otimizar os níveis de estoque com base em diferentes restrições permite compreender o trade-off entre disponibilidade e custo de modo a auxiliar a empresa a escolher a estratégia de estoque que utilize os recursos com eficiência e atenda às expectativas operacionais.
\end{abstract}

Palavras-chave: Manutenção. Estoque. Sobressalentes.

Abstract: This paper presents a simulation model to evaluate the total downtime of a power generation plant due to lack of spare parts as a function of inventory levels of each one of these parts. The model considers items that appear once or twice in the system with reliability configuration such as series and parallel, with or without preventive replacement policy. The stochastic optimization based on Scatter Search metaheuristic is used to set inventory levels in order to minimize the mean total downtime due to lack of parts and respecting the statistical constraints over the total amount spent for the planning horizon. The results indicated that, for example, if the cost constraint of $R \$$ 550,000 for 10 years is increased by $9.1 \%$, the mean total downtime is reduced by $55.9 \%$ (from 617 to 272 hours). Therefore, it demonstrates that optimization of inventory levels based on different constraints allows us to understand the trade-off between availability and cost, which helps the company to choose inventory (stock) strategy that uses resources efficiently and meets operational expectations.

Keywords: Maintenance, Inventory. Spare parts.

\section{INTRODUÇÃO}

Existe, por parte da administração de usinas hidrelétricas, a preocupação em 
assegurar que o nível de disponibilidade da planta seja suficiente para atender à meta anual de geração de energia estipulada pelos órgãos reguladores. Para isso, além das políticas de manutenção e de níveis de redundância dentro do sistema, um fator importante é o dimensionamento dos estoques de peças de reposição (podendo ser equipamentos inteiramente substituíveis), pois:

- existe incerteza na demanda por elementos de reposição, isto é, não se sabe quando um item será requisitado;

- existem elementos com alto custo de aquisição e, como o orçamento é limitado, a quantidade de itens em estoque também fica limitada;

- existe incerteza sobre o lead time de entrega. Entende-se lead time de entrega o intervalo de tempo entre o pedido de compra e o momento da entrega.

Neste sentido, Basten e van Houtum (2014) destacam que a estocagem de peças sobressalentes pode prevenir longos intervalos de indisponibilidade de sistemas técnicos. Alrabghi e Tiwari (2015) enfatizam também a importância que a administração do estoque de peças sobressalentes tem no custo operacional.

O problema do dimensionamento do estoque de peças de reposição ocorre não somente no setor de geração de energia hidrelétrica, mas também em outros segmentos como mineração, petróleo, papel e celulose, siderurgia, e etc. Na prática, esta questão vem sendo abordada com base em (a) tentativa e erro, (b) sugestões de experiências passadas e (c) recomendações de fabricantes. Desta forma, trata-se de um problema real tanto de grandes como de pequenas empresas e há demanda por soluções que contribuam para melhorar a eficiência da alocação de recursos.

O modelo para o dimensionamento da quantidade de peças de reposição deve considerar que existe um trade-off entre a disponibilidade do sistema e o custo de estoque. Ou seja, ao reduzir o valor imobilizado em estoque por meio da diminuição da quantidade de peças de reposição, pode-se aumentar o tempo indisponível do sistema de geração por falta de peças para manutenções. Portanto, dimensionar corretamente o estoque de peças sobressalentes é de vital importância para a lucratividade do negócio.

Dos Santos e Selitto (2016) destacam a importância da estratégia de manutenção estar apoiada em modelos quantitativos para promover o aumento da disponibilidade de um sistema. Neste sentido, no presente trabalho apresenta-se um 
modelo de otimização estocástica da quantidade de peças de reposição com o emprego de planilhas de Excel em conjunto com o software Crystal Ball (aplicativo que funciona dentro do Excel). A escolha desta abordagem possui duas razões principais:

- modelos de simulação de Monte Carlo são mais flexíveis do que modelos analíticos para a representação dos detalhes observados na prática;

- ambos os softwares possuem baixo custo e, deste modo, são acessíveis inclusive às empresas de pequeno porte.

O emprego de planilhas eletrônicas não é comum como ferramenta de implementação de algoritmos, mesmo que tenha um grande potencial e grande quantidade de recursos. No entanto, há exceções como Farasyn et al. (2008), em que um modelo em planilha permitiu a redução de US\$350 milhões em estoques, mesmo sendo um modelo com focos diferentes em relação aos objetivos do deste artigo.

O desenvolvimento do modelo apresentado neste trabalho teve como objetivo definir os níveis de estoque das peças sobressalentes, respeitando restrições estatísticas sobre o orçamento, de modo a minimizar a média da função objetivo (tempo indisponível do sistema decorrente da espera por peças de reposição). Para definir os valores das variáveis de decisão, foi empregada otimização baseada na metaheurística Scatter Search. Desta forma, o desenvolvimento de um modelo em planilha, capaz de resolver um problema real e complexo, deve contribuir para diminuir a lacuna entre trabalhos acadêmicos e a indústria. Preocupação semelhante à observada em autores como Silva Filho et al. (2009).

O artigo estrutura-se do seguinte modo: na seção 2 apresenta-se uma revisão da literatura associada ao tema dimensionamento de peças de reposição, indisponibilidade de sistemas e custos. Na seção 3 detalha-se o desenvolvimento do modelo de simulação e otimização, além de apresentar aplicação numérica. Na seção 4 apresenta-se as principais conclusões.

\section{REVISÃO DE LITERATURA}

Trabalhos como os de Huiskonen (2001) e Sarmah e Moharana (2015) buscam classificar cada peça sobressalente de acordo com seus atributos, onde a maneira na qual a peça é classificada deve orientar sua política de nível de estoque. 
Esta abordagem fornece regras rápidas e estruturadas que ajudam a diminuir o risco de faltar peça e desperdícios gerados por um estoque exagerado. Sua desvantagem é não ter o objetivo de otimizar a disponibilidade de um sistema com base nas variáveis relacionadas aos níveis de estoque e ao orçamento disponível.

Diversos autores se concentram em desenvolver modelos que possam prever a demanda de peças no futuro (DOLGUI; PASHKEVICH, 2008; LENGU et al., 2014; ARMENZONI et al., 2015), mas estes não investigam a relação entre o nível de estoque e indicadores de desempenho operacional do sistema.

Há trabalhos com foco na definição do nível de estoque de cada sobressalente de modo a não exceder um nível de risco de faltar peça predefinido e considerado como aceitável (HAGMARK; PERNU, 2006; ZHU et al., 2015). Trusevych et al. (2014) aperfeiçoou a abordagem de risco predefinido utilizando-se o conceito de Conditional Value-at-Risk para considerar estimativas de possíveis perdas financeiras associadas à falta de peças.

Recentemente nota-se - em autores como Lynch et al. (2013), Bjarnason e Taghipour (2014), Gan et al. (2015), Samal e Pratihar (2015), Sleptchenko e van der Heijden (2016) e Keizer et al. (2017) - a tendência de incluir variáveis de decisão adicionais como níveis de redundância, políticas de manutenção e etc. no desenvolvimento de modelos destinados à definição dos níveis de estoque. Porém, estes trabalhos também não consideram o problema de otimização sob incerteza do nível de peças em estoque para minimizar a média do tempo indisponível respeitando restrições de orçamento.

Smith (2011) e Nakagawa (2011) apontam o uso de cadeias de Markov na análise da relação entre nível de estoque e tempo de indisponibilidade de sistemas. A aplicabilidade destes modelos, porém, é limitada, especialmente devido à premissa restritiva de emprego da distribuição exponencial para modelar a variável tempo até a falha.

Alrabghi et al. (2013) desenvolveram um modelo de otimização de estoques de sobressalentes em que, na função objetivo, aparecem custos de manutenção e indisponibilidade. No entanto, a dificuldade desta abordagem está na dinâmica do custo da indisponibilidade que depende de fatores como condições do mercado e operacionais da própria empresa. Ge et al. (2016), por sua vez, desenvolveram um 
modelo para minimizar os custos de manutenção respeitando as restrições sobre a disponibilidade de suprimento de peças.

Como mostrado na revisão de literatura, os modelos apresentados buscam soluções para problemas pontuais e simplificados quando comparados ao caso real de dimensionamento de sobressalentes.

Uma alternativa para encontrar soluções mais realísticas é pelo emprego do método de Monte Carlo. Neste contexto, o presente artigo busca preencher a lacuna existente na literatura por meio de desenvolvimento e aplicação de um modelo baseado em simulação de Monte Carlo e capaz de relacionar políticas de estoque das peças sobressalentes, política de substituição preventiva, restrição de orçamento e a disponibilidade operacional no nível do sistema. A contribuição do trabalho também se relaciona à apresentação de modelo com a flexibilidade para ser gradualmente modificado para realidades mais complexas, o que nem sempre é possível com o emprego de modelos analíticos.

A solução para o problema envolveu o emprego do algoritmo de otimização Scatter Search, uma metaheurística que explora o espaço de solução pela evolução de um conjunto de pontos de referência (RESENDE et al., 2010) presente na ferramenta OptQuest, um dos módulos do software Crystal Ball utilizado no presente trabalho para realizar as otimizações envolvendo nível de estoque, custo e tempo de indisponibilidade de sistemas.

\section{MODELO: DESENVOLVIMENTO, APLICAÇÃO E DISCUSSÕES}

As principais características do problema envolvendo demanda estocástica de peças, níveis de estoque, indisponibilidade causada pela falta de peças e custos são:

- a duração da vida de cada peça e seu lead time de entrega, que são incertos. Por isto distribuições de probabilidade são utilizadas para a representação da dispersão destas variáveis;

- objetivos conflitantes, ou seja, se o número de peças em estoque for alto, então a probabilidade de faltar alguma peça será baixa, porém, o custo de capital imobilizado será alto. Por outro lado, ao se reduzir o custo de capital imobilizado em estoque aumenta-se a exposição ao risco de falta de peça; 
- existência de restrições orçamentarias em qualquer empresa e expectativas gerenciais sobre o desempenho operacional esperado do sistema.

O modelo de simulação aplicado neste artigo envolveu as etapas apresentadas na Figura 1.

Figura 1 - Metodologia para a apresentação dos resultados

\begin{tabular}{|c|c|c|c|}
\hline Etapa 1 & Etapa 2 & Etapa 3 & Etapa 4 \\
\hline $\begin{array}{c}\text { Modelagem da lógica de } \\
\text { simulação das variáveis } \\
\text { de resposta relacionadas } \\
\text { a indisponibilidade e } \\
\text { custo com o uso dos } \\
\text { software Excel e Crystal } \\
\text { Ball. }\end{array}$ & $\begin{array}{l}\text { Inserção das variáveis } \\
\text { básicas no modelo. } \\
\text { Obs.: Etapa que demanda } \\
\text { estudo prévio do } \\
\text { comportamento estatístico das } \\
\text { variáveis aleatórias. }\end{array}$ & $\begin{array}{l}\text { Otimização do nivel de } \\
\text { estoque de cada peça } \\
\text { para diferentes niveis de } \\
\text { orçamento dadas as } \\
\text { restriçôes e o propósito } \\
\text { de minimizar a média da } \\
\text { funcão objetivo. }\end{array}$ & $\begin{array}{l}\text { Exposição, para uso } \\
\text { gerencial, da relação } \\
\text { entre nível de orçamento } \\
\text { e tempo indisponível por } \\
\text { falta de peça com base } \\
\text { nos resultados das } \\
\text { otimizacóes. }\end{array}$ \\
\hline
\end{tabular}

Na Figura 1, consta uma visão macro da metodologia compreendendo desde a etapa da estruturação da modelagem até a apresentação dos resultados.

$\mathrm{Na}$ etapa 1, elabora-se a estrutura lógica do modelo que utiliza em conjunto os softwares Excel e Crystal Ball para simular a variável de resposta relacionada ao tempo indisponível do sistema por falta de peças (que é a função objetivo da simulação), além da variável relacionada ao custo total de aquisição de peças durante o horizonte de simulação (que é utilizada pela restrição estatística orçamentária nas otimizações).

Na etapa 2, realiza-se a inserção de informações no modelo tais como custos de aquisição de peças, distribuições de probabilidade de duração de vida e lead time de entrega de peças, intervalos de substituição preventiva e a idade atual de cada item em operação.

$\mathrm{Na}$ etapa 3, realiza-se a otimização estocástica (otimização em conjunto com simulação de Monte Carlo) de modo a definir os valores das variáveis de decisão de visando minimizar a média da função objetivo, mas garantindo que o risco de exceder o orçamento definido seja no máximo de $5 \%$.

$\mathrm{Na}$ etapa 4, os resultados são apresentados por meio de tabelas e gráficos, contendo as estatísticas simuladas das variáveis resposta do modelo, a partir das soluções encontradas após realizar as otimizações respeitando diferentes níveis de restrição de orçamento. 
Na Figura 2, mostra-se a lista dos itens de um sistema de geração de energia elétrica considerados no modelo desenvolvido neste artigo.

Figura 2 - Dados de entrada, aba "Planilha resumo"

\begin{tabular}{|c|c|c|c|c|c|c|c|c|c|c|}
\hline 4 & A & B & C & D & $E$ & $\mathrm{~F}$ & G & $\mathrm{H}$ & 1 & J \\
\hline 2 & Tempo de simulação (horas) = & 87.600 & \multicolumn{2}{|c|}{$\begin{array}{c}\text { Idade atual do } \\
\text { item (horas) }\end{array}$} & \multicolumn{2}{|c|}{$\begin{array}{c}\text { Modelagem do tempo até } \\
\text { a falha, parâmetros da } \\
\text { distribuição Weibull }\end{array}$} & \multirow{2}{*}{$\begin{array}{l}\text { Intervalo } \\
\text { de } \\
\text { preventiva } \\
\text { (horas) }\end{array}$} & \multicolumn{2}{|c|}{$\begin{array}{l}\text { Modelagem do lead time de } \\
\text { entrega, parâmetros da } \\
\text { distribuição lognormal }\end{array}$} & \multirow{2}{*}{$\begin{array}{c}\text { Custo } \\
\text { unitário do } \\
\text { item (R\$) }\end{array}$} \\
\hline 4 & Itens & Classificação & A & B & $\begin{array}{c}\text { Eta } \\
\text { (horas) }\end{array}$ & Beta & & $\begin{array}{l}\text { Média } \\
\text { (horas) }\end{array}$ & $\begin{array}{c}\text { Desvio padrão } \\
\text { (horas) }\end{array}$ & \\
\hline 5 & Anel coletor da excitação & Item único & 0 & & 27.156 & 1,1 & 99.999 & 360 & 72 & $\mathrm{R} \$ 5.100$ \\
\hline 6 & Bucha - TE & Item único & 0 & & 264.552 & 2,1 & 99.999 & 504 & 50 & $\mathrm{R} \$ 11.110$ \\
\hline 7 & Câmera de extinção & Item único & 0 & & 130.524 & 1,9 & 99.999 & 480 & 96 & $\mathrm{R} \$ 12.330$ \\
\hline 8 & Capacitor - CS & Item único & 0 & & 86.724 & 1,8 & 99.999 & 240 & 72 & $\mathrm{R} \$ 10.910$ \\
\hline 9 & Disjuntor de sincronismo & Item único & 0 & & 86.286 & 2 & 99.999 & 336 & 33 & $\mathrm{R} \$ 19.930$ \\
\hline 10 & Disjuntor principal & Item único & 0 & & 91.980 & 2,1 & 99.999 & 360 & 72 & $\mathrm{R} \$ 17.880$ \\
\hline 11 & Enrolamento - TE & Item único & 0 & & 261.924 & 1 & 99.999 & 672 & 67 & $\mathrm{R} \$ 53.010$ \\
\hline 12 & Filtro autolimpante & Dois em paralelo & 0 & 0 & 6.118 & 2,7 & 4.380 & 168 & 16 & $\mathrm{R} \$ 295$ \\
\hline 13 & Filtro cesto & Dois em paralelo & 0 & 0 & 6.342 & 2,9 & 4.380 & 72 & 14 & $\mathrm{R} \$ 115$ \\
\hline 14 & Isolador & Dois em série & 0 & 0 & 253.164 & 1,5 & 99.999 & 168 & 16 & $\mathrm{R} \$ 4.990$ \\
\hline 15 & Mancal guia & Item único & 0 & & 45.552 & 2,9 & 35.000 & 240 & 48 & $R \$ 5.700$ \\
\hline 16 & Núcleo - TE & Item único & 0 & & 279.444 & 2,2 & 99.999 & 720 & 144 & $\mathrm{R} \$ 51.050$ \\
\hline 17 & Para-raios & Dois em série & 0 & 0 & 42.924 & 1 & 99.999 & 336 & 33 & $\mathrm{R} \$ 10.090$ \\
\hline 18 & Relé de bloqueio & Dois em série & 0 & 0 & 28.908 & 1,1 & 99.999 & 48 & 14 & $\mathrm{R} \$ 1.300$ \\
\hline 19 & Relé de gás - TE & Item único & 0 & & 88.476 & 1 & 99.999 & 72 & 7 & $\mathrm{R} \$ 900$ \\
\hline 20 & Relé de proteção & Dois em série & 0 & 0 & 28.032 & 1 & 99.999 & 72 & 7 & $\mathrm{R} \$ 1.200$ \\
\hline 21 & Resistor de aterramento & Item único & 0 & & 88.038 & 2,1 & 99.999 & 48 & 10 & $\mathrm{R} \$ 13.100$ \\
\hline 22 & Sapata & Dois em série & 0 & 0 & 27.156 & 3,2 & 17.520 & 120 & 24 & $\mathrm{R} \$ 5.110$ \\
\hline 23 & Sensor de pressão & Item único & 0 & & 121.764 & 2,3 & 99.999 & 480 & 96 & $\mathrm{R} \$ 13.150$ \\
\hline 24 & Transformador de corrente & Item único & 0 & & 141.036 & 2,1 & 99.999 & 504 & 100 & $\mathrm{R} \$ 22.150$ \\
\hline 25 & Transformador de potencial & Item único & 0 & & 127.020 & 2,5 & 99.999 & 480 & 144 & $\mathrm{R} \$ 21.200$ \\
\hline 26 & Vedação de eixo da turbina & Item único & 0 & & 124.392 & 3,2 & 99.999 & 120 & 36 & $\mathrm{R} \$ 5.910$ \\
\hline
\end{tabular}

14 4 M| Transformador de corrente Transformador de potencial / Vedação de eixo da turbina Planilha resumo |1 4

Fonte: Elaboração própria

Na célula B2 é apresentado o horizonte de tempo de simulação, que é igual a 87.600 horas (10 anos de operação).

$\mathrm{Na}$ respectiva linha, onde se encontra o nome do item, observa-se também os parâmetros de entrada, como os utilizados para a modelagem do tempo até a falha, lead time de entrega, custo unitário de aquisição do item, intervalo de substituição preventiva e idade atual do item.

As colunas presentes na planilha da Figura 2, cujo conteúdo pode ser observado a partir da linha 5 , são descritas a seguir:

- na coluna A encontra-se a lista dos nomes dos itens em questão;

- na coluna B cada item é classificado em relação à sua posição dentro da configuração de confiabilidade do sistema. Tais classificações são utilizadas para escolher a modelagem necessária para simular a relação que o nível de estoque (e eventualmente também o intervalo de substituições preventivas) 
tem com a indisponibilidade por falta peças e o custo total de aquisição do item. Estas classificações são:

o item único: ocorre quando o item aparece somente uma vez no sistema (apenas uma localização física) e sua falta provoca necessariamente a indisponibilidade de todo o sistema. Exemplo: Bucha-TE.

o dois em série: ocorre quando o item aparece duas vezes no sistema, sendo que a falha de um deles torna o sistema indisponível. O mesmo estoque de sobressalentes é utilizado quando um dos dois itens precisar ser substituído. Exemplo: Relé de proteção.

o dois em paralelo: ocorre quanto o item também aparece duas vezes no sistema, mas a falha de apenas um deles não causa a falha do sistema. O mesmo estoque pode ser utilizado na substituição de qualquer um dos dois itens. Exemplo: Filtro autolimpante.

- a coluna $\mathbf{C}$ contém a idade atual do item que já se encontra em operação. Para itens com a descrição "Dois em série" ou "Dois em paralelo", a coluna D permite que seja inserida uma segunda idade respectiva a um segundo item do mesmo tipo em operação.

- nas colunas E e F encontram-se, respectivamente, os parâmetros Eta (escala) e Beta (forma) da distribuição Weibull empregada na modelagem de vida de cada um dos itens. As principais distribuições de probabilidade para modelagem do tempo de vida de componentes são Weibull, exponencial, normal e lognormal e, dentre estas, a distribuição Weibull é aquela mais usada devido à sua flexibilidade para gerar resultados próximos das demais distribuições (KECECIOGLU, 2002; NELSON, 2005; EBELING, 2010; BIROLINI, 2014);

- na coluna $\mathbf{G}$ encontram-se os intervalos de substituição preventiva. Se o valor for superior ao horizonte de simulação, significa que não é feita a substituição preventiva deste item;

- nas colunas H e I encontram-se, respectivamente, os parâmetros de média e desvio padrão da distribuição lognormal utilizada para modelar o lead time de entrega. Neste caso, ambos os parâmetros estão na unidade de horas, ou seja, não se referem a valores logarítmicos. A característica de assimetria à 
direita da distribuição lognormal - uma vez que ocorrem casos em que o tempo pode ser muito superior ao esperado e, por isso, a média é maior que a moda (KECECIOGLU, 2002; EBELING, 2010) - motivou o emprego desta distribuição;

- na coluna $\mathbf{J}$ mostra-se o custo unitário de cada um dos itens.

A partir destas considerações, desenvolveu-se o modelo. Na seção 3.1 é apresentado o modelo desenvolvido para itens do tipo "Item único" e na seção 3.2 para itens do tipo "Dois em paralelo" e "Dois em série". O propósito destes modelos foi simular, para cada classificação de item existente na coluna B da Figura 2, a indisponibilidade decorrente da falta de peças em estoque e o custo de aquisição destas peças em função do seu nível de estoque.

$\mathrm{Na}$ seção 3.3 é discutido o modelo agregado que considera todos os itens listados. Este modelo agregado foi utilizado para simular os valores totais de indisponibilidade e custo para realizar a otimização estocástica dos níveis de estoque considerando-se a restrição de orçamento.

\subsection{Modelo para itens que aparecem apenas uma vez no sistema}

Na sequência é considerado o caso de um componente que aparece em apenas uma localização dentro do sistema e, cuja falha causa também a falha do sistema, configuração descrita como "Item único".

$\mathrm{Na}$ Figura 3, mostra-se a planilha com o modelo utilizado para simular os impactos da política de estoque do item denominado mancal guia no custo e indisponibilidade.

O modelo de simulação pertinente ao mancal guia encontra-se na aba de igual nome. Destaca-se que a compra dos itens para estoque é realizada de forma individual e não em lotes. As premissas básicas na Figura 3, que fazem referência aos mesmos parâmetros apresentados na aba "Planilha resumo" indicada na Figura 2, são:

- horizonte de simulação (Célula C2);

- idade atual do item (Célula C6);

- parâmetros Beta (Célula E4) e Eta (Célula E5) da distribuição Weibull utilizada na modelagem do tempo até a falha; 
- intervalo de substituição preventiva (Célula F7);

- média (Célula G4) e desvio padrão (Célula G5) da distribuição lognormal utilizada na modelagem do lead time de entrega.

Figura 3 - Modelagem da indisponibilidade por falta de peças do mancal guia

\begin{tabular}{|c|c|c|c|c|c|c|c|}
\hline 4 & A & B & C & D & $\mathrm{E}$ & $\mathrm{F}$ & G \\
\hline 1 & \multicolumn{7}{|l|}{ MANCAL GUIA } \\
\hline 2 & \multicolumn{2}{|c|}{ Tempo de simulação (horas) = } & 87.600 & \multicolumn{2}{|c|}{ Modelagem do tempo até a falha } & \multicolumn{2}{|c|}{ Modelagem do lead time de entrega } \\
\hline 3 & \multicolumn{2}{|c|}{ Tempo indisponível por espera (horas) = } & 0 & \multicolumn{2}{|c|}{ Distribuição Weibull } & \multicolumn{2}{|c|}{ Distribuição lognormal } \\
\hline 4 & \multicolumn{2}{|c|}{ Nível máximo de estoque $=$} & 2 & Beta $=$ & 2,9 & Média (horas) = & 240 \\
\hline 5 & \multicolumn{2}{|c|}{ Início do estoque (horas) = } & 0 & Eta (horas) $=$ & 45.552 & Desvio padrão (horas) $=$ & 48 \\
\hline 6 & \multicolumn{2}{|c|}{ Idade atual do item (horas) = } & 0 & & & & \\
\hline 7 & \multicolumn{2}{|c|}{ Valor unitário do item = } & $\mathrm{R} \$ 5.700$ & \multicolumn{2}{|c|}{ Intervalo de preventiva (horas) $=$} & 35.000 & \\
\hline 8 & \multicolumn{2}{|c|}{ Valor gasto com aquisição do item = } & $\mathrm{R} \$ 22.800$ & & & & \\
\hline \multicolumn{8}{|l|}{9} \\
\hline 10 & $\begin{array}{l}\text { Número do } \\
\text { evento }\end{array}$ & $\begin{array}{l}\text { Tempo até a falha } \\
\text { simulado (horas) }\end{array}$ & $\begin{array}{c}\text { Início da manutenção } \\
\text { ou espera pelo item } \\
\text { (horas) }\end{array}$ & $\begin{array}{c}\text { Lead time } \\
\text { simulado do } \\
\text { pedido (horas) }\end{array}$ & $\begin{array}{l}\text { Chegada da item } \\
\text { pedido (horas) }\end{array}$ & $\begin{array}{c}\text { Número de itens no } \\
\text { estoque antes da } \\
\text { manutenção }\end{array}$ & $\begin{array}{c}\begin{array}{c}\text { Tempo indisponível } \\
\text { devido espera pelo } \\
\text { item (horas) }\end{array} \\
\end{array}$ \\
\hline 12 & 1 & $46.880,1$ & $35.000,0$ & 232,4 & $35.000,0$ & 2 & 0,0 \\
\hline 13 & 2 & $61.399,1$ & $70.000,0$ & 292,8 & $70.000,0$ & 2 & 0,0 \\
\hline 14 & 3 & $20.334,8$ & $90.334,8$ & 153,0 & $90.487,8$ & 2 & 0,0 \\
\hline 15 & 4 & $15.587,4$ & $105.922,2$ & 321,4 & $106.243,6$ & 2 & 0,0 \\
\hline 16 & 5 & $37.994,4$ & $140.922,2$ & 250,4 & $140.922,2$ & 2 & 0,0 \\
\hline 17 & 6 & $65.572,0$ & $175.922,2$ & 214,7 & $175.922,2$ & 2 & 0,0 \\
\hline 18 & 7 & $53.086,6$ & $210.922,2$ & 285,2 & $210.922,2$ & 2 & 0,0 \\
\hline 19 & 8 & $30.622,2$ & $241.544,4$ & 146,9 & $241.691,2$ & 2 & 0,0 \\
\hline
\end{tabular}

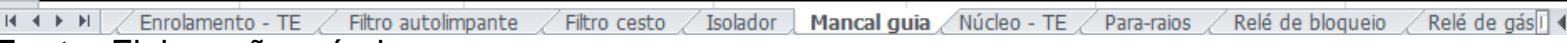
Fonte: Elaboração própria

As variáveis de decisão, que também fazem referência a aba "Planilha resumo" como explicado posteriormente na seção 3.3, utilizadas são:

- nível máximo de estoque (Célula C4);

- início do estoque (Célula C5). Indica quando o estoque deste item pode passar a ser considerado maior do que zero. Esta variável pode ser utilizada especialmente para itens de longa vida esperada que apresentem degradação;

As explicações sobre as colunas cujos títulos se encontram na linha 10 são:

- coluna A: representa o número da linha da tabela de cálculo (ou seja, o número do evento de demanda simulado naquela linha);

- coluna B: utiliza a função CB.Weibull(<Parâmetros>) para simular um tempo até a falha do item de acordo com a distribuição Weibull. Para esta função, reservada ao Crystal Ball, o primeiro argumento refere-se ao parâmetro de localização (que pode ser omitido se for igual a zero), o segundo argumento refere-se ao parâmetro de escala (Eta) e o terceiro indica o parâmetro de forma (Beta); 
- coluna C: é responsável por calcular, com base na aleatoriedade, o momento da necessidade de uma peça para a manutenção;

- coluna D: utiliza a função CB.Lognormal(<Parâmetros>) para simular o lead time de entrega. Para esta função, o primeiro e segundo argumentos se referem-se, respectivamente, à média e ao desvio padrão da distribuição na mesma unidade do valor simulado (a função CB.Lognormal não utilizada parâmetros em escala logarítmica);

- coluna E: calcula quando o item, indicado na coluna A, chegou de fato ao estoque;

- coluna F: calcula quantas peças existem no estoque antes realizar a manutenção simulada na mesma linha;

- coluna G: calcula o tempo indisponível decorrente da espera pelo item faltante.

A linha 11 da planilha da Figura 3 (interprete como a linha número zero da coluna) foi deixada em branco por questões de facilitação na exposição das equações. No Quadro 1 mostra-se as equações utilizadas na primeira linha (ou seja, linha número 12 da planilha) de cada coluna descrita.

Quadro 1 - Equações presentes na décima segunda linha do modelo apresentado na Figura 3.

\begin{tabular}{|c|c|}
\hline Coluna & Equação da primeira linha \\
\hline $\mathrm{B}$ & $=$ CB.Weibull $(; \$ E \$ 5 ; \$ E \$ 4 ; \$ C \$ 6)$ \\
\hline $\mathrm{C}$ & $=\mathrm{C} 11+\mathrm{G} 11+\mathrm{SE}(\mathrm{B} 12<\$ F \$ 7 ; \mathrm{B} 12 ; \$ \mathrm{~F} \$ 7)$ \\
\hline $\mathrm{D}$ & $=$ CB.Lognormal $(\$ G \$ 4 ; \$ G \$ 5)$ \\
\hline $\mathrm{E}$ & $=\mathrm{SE}(\mathrm{B} 12>\$ \mathrm{~F} \$ 7 ; \mathrm{C} 12 ; \mathrm{MIINIMO}(\mathrm{C} 12+\mathrm{D} 12 ; \mathrm{C} 11+\mathrm{G} 11+\$ \mathrm{~F} \$ 7))$ \\
\hline $\mathrm{F}$ & $=(-\mathrm{A} 12+1)+\mathrm{SE}(\$ \mathrm{C} \$ 5>\mathrm{C} 12 ; 0 ; \$ C \$ 4)+\mathrm{CONT} . \mathrm{SE}(\$ \mathrm{E} \$ 12: \mathrm{E} 12 ; "<" \& \mathrm{C} 12)$ \\
\hline G & $\begin{array}{l}=\mathrm{SE}(\mathrm{E}(\mathrm{C} 12<\$ C \$ 2 ; \mathrm{F} 12=0) ; \mathrm{MENOR}(\$ \mathrm{E} \$ 12: \mathrm{E} 12 ; \mathrm{A} 12-\$ \mathrm{C} \$ 4 * \mathrm{SE}(\$ \mathrm{C} \$ 5>\mathrm{C} 12 ; \\
0 ; 1))-\mathrm{C} 12 ; 0)\end{array}$ \\
\hline
\end{tabular}

Fonte: Elaboração própria

Entre todas as equações apresentadas no Quadro 1, apenas a B exige modificação antes de ser copiada para as linhas seguintes. O quarto parâmetro da equação =CB.Weibull(; $\$ E \$ 5 ; \$ E \$ 4 ; \$ C \$ 6)$, ou seja $\$ C \$ 6$, refere-se à idade atual do item em operação e deve ser removido para as linhas seguintes.

O número de linhas de simulação deve garantir que o período total de simulação (célula C2) seja menor do que o valor da última linha da coluna que 
representa o momento do início da manutenção ou espera pelo item (na Coluna C). No caso do mancal guia foram utilizadas oito linhas.

As Equações 1, 2, 3 e 4 equivalem, respectivamente, às equações das colunas C, E, F e G indicadas no Quadro 1. A função $f_{c}$ representa a coluna c, $f_{E}$ a coluna $\mathrm{E}$ e assim sucessivamente.

$$
\begin{aligned}
& f_{C}(n)=f_{C}(n-1)+f_{G}(n-1)+f_{C 1}(n) \\
& f_{C 1}(n)=\left\{\begin{array}{cc}
T F_{n} & \text { se } T F_{n}<P \\
P & \text { se } T F_{n} \geq P
\end{array}\right. \\
& f_{E}(n)=\left\{\begin{array}{cc}
f_{C}(n) & \text { se } T F_{n}>P \\
\min \left(f_{C}(n)+T L_{n} ; f_{C}(n-1)+f_{G}(n-1)+P\right) & \text { se } T F_{n} \leq P
\end{array}\right. \\
& f_{F}(n)=(-n+1)+f_{F 1}(n)+\sum_{i=1}^{n} \sum_{f_{E}(i)<f_{C}(n)} 1 \\
& f_{F 1}(n)= \begin{cases}0 & \text { se } I>f_{C}(n) \\
M & \text { se } I \leq f_{C}(n)\end{cases} \\
& f_{G}(n)=\left\{\begin{array}{cc}
f_{g 1}(n)-f_{C}(n) & \text { se } f_{C}(n)<H \& f_{F}(n)=0 \\
0 & \text { caso contrário }
\end{array}\right. \\
& f_{G 1}(n)=\left\{\begin{array}{cc}
f_{E}(n) & \text { se } f_{C}(n)<I \\
f_{E}(n-M) & \text { se } f_{C}(n) \geq I
\end{array}\right.
\end{aligned}
$$

onde $\mathrm{n}$ indica o número do evento de demanda por peça, $T F_{n}$ o tempo até a falha simulado para o evento $\mathrm{n}, T L_{n}$ o tempo de lead time de entrega da peça demandada após o evento n, P o intervalo de substituição preventiva, I o momento de início do estoque, $\mathrm{M}$ o nível máximo de estoque e $\mathrm{H}$ o horizonte de simulação. Destaca-se que a simulação de $T F_{1}$ deve ser truncada inferiormente na idade do item atualmente em operação, também que $f_{c}(0)$ e $f_{G}(0)$ são igual a zero.

As duas variáveis de resposta deste modelo são:

- tempo total indisponível devido à espera por peças faltantes (no caso o mancal guia) em estoque (Célula C3). A equação utilizada na planilha é a =SOMA(G12:G19) que é responsável por somar todo o tempo indisponível devido à falta de peças em estoque, equivalente à expressão:

Tempo indisponível por espera $=\sum_{i=1}^{N} f_{G}(i)$

onde $\mathrm{N}$ representa o número total de eventos simulados. 
- valor total gasto simulado com a aquisição de peças (Célula C8). A equação utilizada na planilha é a $=(\operatorname{CONT} . \operatorname{SE}(\mathrm{C} 12: \mathrm{C} 19 ; "<=" \& C 2)+\mathrm{C} 4){ }^{\star} \mathrm{C} 7$ que é responsável por somar todo o custo com aquisição de peças, o que inclui estoque inicial e demanda simulada, equivalente à expressão:

Valor gasto com aquisição do item $=\left(M+\sum_{i=1}^{N} \sum_{f_{C}(i) \leq H} 1\right) * C$

onde $\mathrm{C}$ representa o custo unitário de aquisição da peça.

É importante enfatizar que as equações presentes nas células C3 e C8 da planilha do Excel devem ser modificadas para itens com número de linhas de simulação maior do que 8.

A análise de todas as variáveis de resposta neste trabalho considerou 1.000 simulações e se baseou em um horizonte de análise de 10 anos (87.600 horas). $\mathrm{Na}$ Figura 4 destaca-se o histograma gerado pela simulação do tempo indisponível por espera devido à falta de mancal guia (célula C3) para um cenário de inexistência de estoque de peças sobressalentes.

Figura 4 - Tempo indisponível por espera do mancal guia

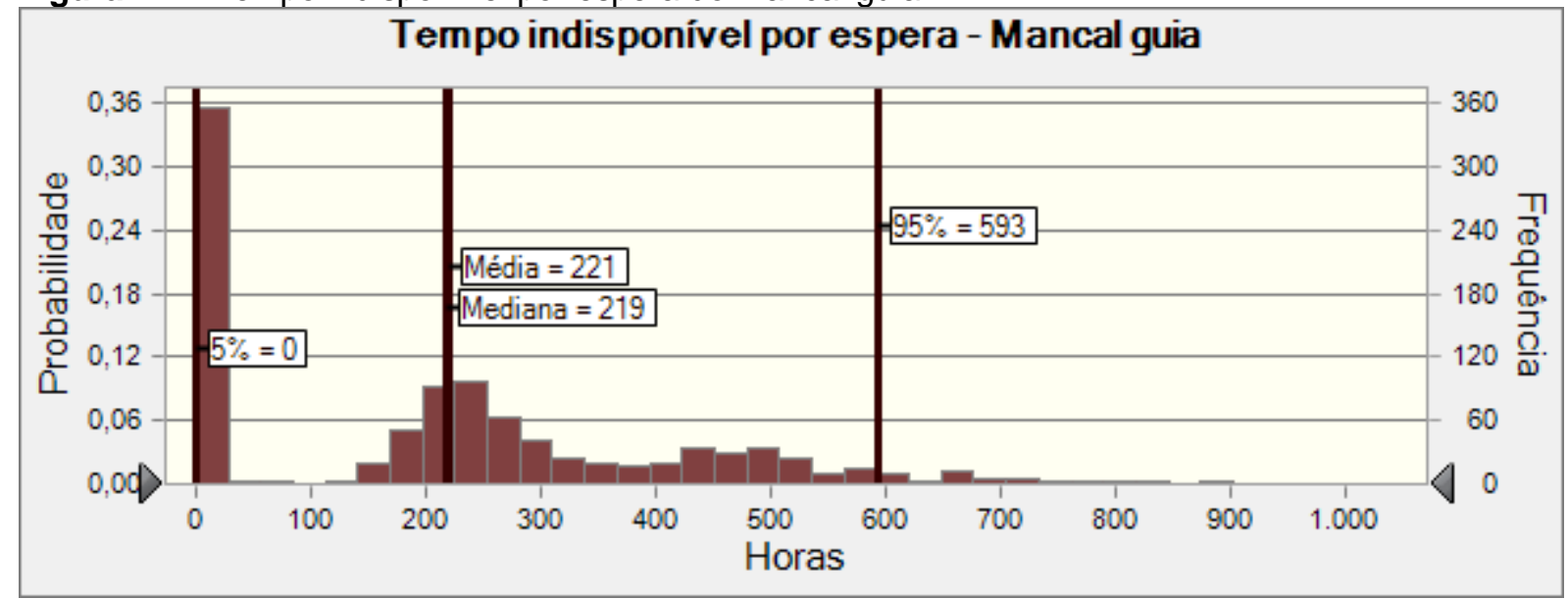

Fonte: Elaboração própria com o auxílio do software Crystal Ball

O valor que aparece com maior frequência em mais de 35\% das simulações, é o de zero horas indisponíveis por espera do item mancal e, por consequência, o percentil 5 também é igual a zero horas. Porém, a média de 221 horas e mediana igual a 219 horas, percentil 95 igual a 593 horas e valor máximo de 1.042 horas indicam que o estoque do mancal guia igual a zero pode causar muitas horas de indisponibilidade no sistema. Ressalta-se que ao realizar uma simulação 
considerando-se o nível de estoque máximo igual a uma peça, não se verificou indisponibilidade por falta de sobressalentes.

Novamente considerando a simulação para um nível de estoque do mancal guia igual a zero item, na Figura 5 encontra-se histograma da simulação do valor de desembolso com a aquisição durante o horizonte de análise.

Figura 5 - Valor gasto aquisição de mancais guia.

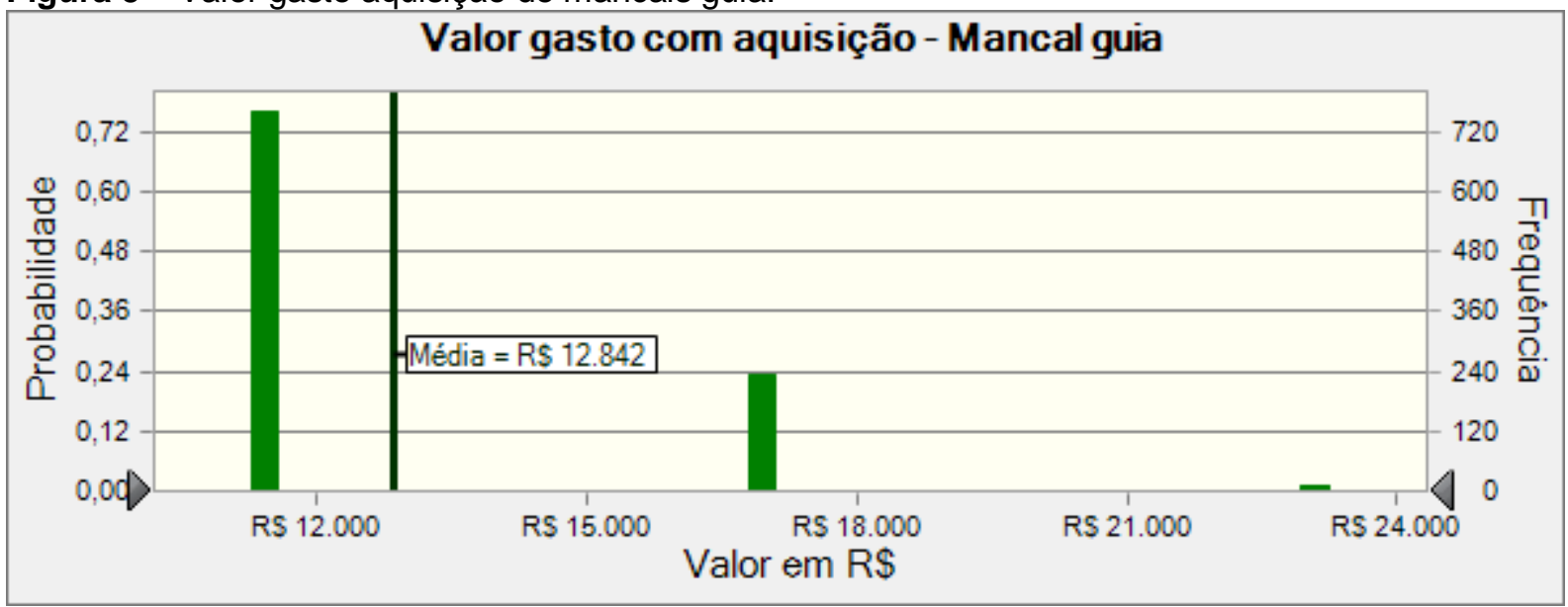

Fonte: Elaboração própria com o auxílio do software Crystal Ball

O valor de R\$ 11.400 aparece em 75,7\% das simulações e é compatível com duas peças adquiridas com custo de $R \$ 5.700$ cada. Os valores de $R \$ 17.100$ e $R \$$ 22.800 aparecem em, respectivamente, $23,3 \%$ e $1,0 \%$ das simulações. As proporções indicadas resultam em uma média de aproximadamente $\mathrm{R} \$ 12.842$. Assim, mesmo que o custo de aquisição do mancal guia seja assumido como determinístico, o valor total gasto com a aquisição de mancais será uma variável aleatória, pois a demanda pelo item é uma variável aleatória. Na simulação refeita para nível de estoque máximo de uma peça, a média observada foi de $\mathrm{R} \$ 18.648$.

Uma vantagem prática deste modelo de simulação é a possibilidade do analista de inserir aleatoriedade na variável custo de aquisição dos itens de maneira mais sofisticada (como utilizar séries temporais para simular a inflação setorial).

\subsection{Modelo para itens que aparecem duas vezes no sistema}

Nesta seção forem desenvolvidos modelos para itens de estoque que aparecem duas vezes no sistema, ou seja, inclui a classificação "Dois em paralelo" e "Dois em série". 
O caso de dois elementos em paralelo foi considerado primeiro. Na Figura 6 encontra-se parte da aba empregada na modelagem do tempo indisponível do sistema decorrente da falta de filtro autolimpante em estoque.

Figura 6 - Modelagem da indisponibilidade por falta de peças do filtro autolimpante

Figura 6a: Parte I

\begin{tabular}{|c|c|c|c|c|c|c|c|}
\hline 4 & A & B & C & D & $\mathrm{E}$ & $\mathrm{F}$ & G \\
\hline 1. & \multicolumn{7}{|c|}{ FILTRO AUTOLIMPANTE } \\
\hline 2 & \multicolumn{2}{|c|}{ Tempo de simulação (horas) = } & 87.600 & \multicolumn{2}{|c|}{ Modelagem do tempo até a falha } & \multicolumn{2}{|c|}{ Modelagem do lead time de entrega } \\
\hline 3 & \multicolumn{2}{|c|}{ Tempo indisponível por espera (horas) = } & 0 & \multicolumn{2}{|c|}{ Distribuição Weibull } & \multicolumn{2}{|c|}{ Distribuição lognormal } \\
\hline 4 & \multicolumn{2}{|c|}{ Nivel máximo de estoque = } & 2 & Beta $=$ & 2,7 & Média (horas) = & 168 \\
\hline 5 & \multicolumn{2}{|c|}{ Início do estoque (horas) = } & 0 & Eta (horas) $=$ & 6.118 & Desvio padrão (horas) = & 16 \\
\hline 6 & \multicolumn{2}{|c|}{ Idade atual do item A (horas) = } & 0 & & & & \\
\hline 7 & \multicolumn{2}{|c|}{ Idade atual do item $\mathrm{B}$ (horas) = } & 0 & \multicolumn{2}{|c|}{ Intervalo de preventiva (horas) $=$} & 4.380 & \\
\hline 8 & \multicolumn{2}{|c|}{ Valor unitário do item $(\mathrm{R} \$)=$} & $\mathrm{R} \$ 295$ & & & & \\
\hline 9 & \multicolumn{2}{|c|}{ Valor gasto com aquisição do item $(\mathrm{R} \$$ ) = } & $\mathrm{R} \$ 13.275,00$ & & & & \\
\hline \multicolumn{8}{|c|}{10} \\
\hline 11. & Número do evento & $\begin{array}{c}\text { Tempo até a falha } \\
\text { simulado do item } \\
\text { A (horas) }\end{array}$ & $\begin{array}{c}\text { Tempo até a falha } \\
\text { simulado do item } \\
\text { B (horas) }\end{array}$ & $\begin{array}{c}\text { Tempo até a } \\
\text { manutenção } \\
\text { acumulado do item } \\
\text { A (horas) } \\
\end{array}$ & $\begin{array}{c}\text { Tempo até a } \\
\text { manutenção } \\
\text { acumulado do item B } \\
\text { (horas) }\end{array}$ & $\begin{array}{c}\text { Início da manutenção } \\
\text { ou da espera pelo item } \\
\text { (horas) }\end{array}$ & $\begin{array}{c}\text { Qual item } \\
\text { que passou } \\
\text { por } \\
\text { manutenção? }\end{array}$ \\
\hline 12 & & & & & & & \\
\hline 13 & 1 & 9664,8 & 6859,4 & $4.380,0$ & $4.380,0$ & $4.380,0$ & A \\
\hline 14 & 2 & 6806,4 & 4561,4 & $8.760,0$ & $8.760,0$ & $4.380,0$ & B \\
\hline 15 & 3 & 6601,6 & 4345,1 & $13.140,0$ & $13.105,1$ & $8.760,0$ & A \\
\hline 141 & 1 A Filtro autolir & npante Filtro cesto & Isolador / Manca & I guia Núcleo-TE & Para-raios / Relé de blc & Relé de gán 4 & \\
\hline
\end{tabular}

Figura 6b: Parte II

\begin{tabular}{|c|c|c|c|c|c|c|c|c|}
\hline 4 & H & I & J & K & L & M & $\mathrm{N}$ & 0 \\
\hline 11. & $\begin{array}{l}\text { Início corrigido da } \\
\text { manutenção ou } \\
\text { espera pelo item } \\
\text { (horas) }\end{array}$ & $\begin{array}{c}\text { Lead time } \\
\text { simulado do } \\
\text { pedido (horas) }\end{array}$ & $\begin{array}{c}\text { Consulta do } \\
\text { tempo até a falha } \\
\text { simulado (horas) }\end{array}$ & $\begin{array}{l}\text { Momento previsto } \\
\text { para próxima } \\
\text { preventiva (horas) }\end{array}$ & $\begin{array}{l}\text { Chegada da } \\
\text { peça pedida } \\
\text { (horas) }\end{array}$ & $\begin{array}{l}\text { Número de peças no } \\
\text { estoque antes da } \\
\text { manutenção (horas) }\end{array}$ & $\begin{array}{l}\text { Tempo de } \\
\text { espera por } \\
\text { peça (horas) }\end{array}$ & $\begin{array}{c}\text { Tempo indisponível } \\
\text { devido espera por } \\
\text { peça (horas) }\end{array}$ \\
\hline 13 & $4.380,0$ & 154,0 & $9.664,8$ & $4.380,0$ & $4.380,0$ & 2 & 0,0 & 0,0 \\
\hline 14 & $4.380,0$ & 162,2 & $6.859,4$ & $4.380,0$ & $4.380,0$ & 2 & 0,0 & 0,0 \\
\hline 15 & $8.760,0$ & 148,4 & $6.806,4$ & $8.760,0$ & $8.760,0$ & 2 & 0,0 & 0,0 \\
\hline
\end{tabular}

Fonte: Elaboração própria

As premissas observadas na parte superior da Figura 6a fazem referência aos parâmetros de entrada já indicados na aba representada pela Figura 2. O que diferencia o conjunto das premissas deste modelo em relação ao conjunto da seção 3.1 é a existência de duas idades atuais para itens indicados genericamente como A e $B$, respectivamente, nas células C6 e C7 da Figura 6a. Esta diferença se justifica pelo fato do modelo considerar o estoque de um item que aparece duas vezes na representação do sistema.

As descrições das colunas cujos títulos se encontram na linha 11 da Figura 6 são:

- coluna A: representa o número do evento de demanda simulado naquela linha; 
- colunas B e C: são utilizadas para simular o tempo até falha do item A e B, respectivamente;

- coluna D e E: calcula o tempo acumulado das colunas B e C, respectivamente;

- coluna F: é utilizada para calcular o momento de início da manutenção ou espera pelo item. A primeira linha indica a primeira manutenção do item modelado na respectiva aba, a segunda linha indica a segunda manutenção e assim sucessivamente, independentemente de ser o item A ou B;

- coluna G: uma vez que a coluna F não faz distinção do item que passou por manutenção, a coluna $\mathrm{G}$ indica qual foi este item;

- coluna H: calcula o momento de início da manutenção ou da espera pelo item corrigido pelo tempo que a respectiva localização do item no sistema ficou indisponível por falta de peça;

- coluna I: simula o lead time de entrega do pedido;

- coluna J: identifica qual foi o tempo até a falha simulado para a manutenção em questão da coluna F;

- coluna K: apresenta o momento previsto para a preventiva, mesmo sabendo que a manutenção indicada naquela linha pode ser uma falha;

- coluna L: a primeira linha indica o tempo de chegada da primeira peça pedida, a segunda linha indica a segunda peça pedida e, assim, sucessivamente;

- coluna M: apresenta o número de peças no estoque antes da manutenção indicada na respectiva linha;

- coluna N: calcula o tempo de espera por peça;

- coluna O: calcula o tempo indisponível de um sistema redundante devido a espera por peça. Ou seja, a falta de uma peça em estoque só tornará o sistema indisponível se o outro item redundante também estiver indisponível. No Quadro 2, encontram-se as equações utilizadas na primeira linha das colunas (linha 13 da planilha da Figura 6). 
Quadro 2 - Equações presentes da décima terceira linha do modelo da Figura 6

\begin{tabular}{|c|c|}
\hline Coluna & Equações da primeira linha \\
\hline $\mathrm{B}$ & $=$ CB.Weibull $(; \mathrm{E} \$ 5 ; \$ E \$ 4 ; \$ C \$ 6)$ \\
\hline C & $=$ CB.Weibull(; $\$ E \$ 5 ; \$ E \$ 4 ; \$ C \$ 7)$ \\
\hline $\mathrm{D}$ & $=\mathrm{SE}(\mathrm{B} 13>\$ \mathrm{~F} \$ 7 ; \$ \mathrm{~F} \$ 7 ; \mathrm{B} 13)+\mathrm{D} 12$ \\
\hline $\mathrm{E}$ & $=\mathrm{SE}(\mathrm{C} 13>\$ \mathrm{~F} \$ 7 ; \$ \mathrm{~F} \$ 7 ; \mathrm{C} 13)+\mathrm{E} 12+0,0001$ \\
\hline $\mathrm{F}$ & $=\mathrm{MENOR}(\$ \mathrm{D} \$ 13: \$ \mathrm{E} \$ 67 ; \mathrm{A} 13)$ \\
\hline G & $=$ SE(CONT.SE $(\$ D \$ 13: \$ D \$ 62 ; "=" \& F 13)=1 ; " A " ; " B ")$ \\
\hline $\mathrm{H}$ & $=F 13+$ SOMASE $(\$ G \$ 12: G 12 ; "=" \& G 13 ; \$ N \$ 12: N 12)$ \\
\hline $\mathrm{I}$ & $=$ CB.Lognormal $(\$ G \$ 4 ; \$ G \$ 5)$ \\
\hline $\mathrm{J}$ & $\begin{array}{l}=\mathrm{DESLOC}(\$ B \$ 13 ; \mathrm{SEERRO}(\mathrm{CORRESP}(\mathrm{F} 13 ; \$ \mathrm{D} \$ 13 ; \$ \mathrm{D} \$ 62 ; 0) ; \mathrm{CORRESP}( \\
\mathrm{F} 13 ; \$ \mathrm{E} \$ 13: \$ \mathrm{E} \$ 62 ; 0))-1 ; \mathrm{SE}(\mathrm{CONT} . \mathrm{SE}(\$ \mathrm{D} \$ 13: \$ \mathrm{D} \$ 2 ; "=" \& \mathrm{~F} 13)=1 ; 1 ; 2)-1)\end{array}$ \\
\hline $\mathrm{K}$ & $=\mathrm{SE}(\mathrm{J} 13>\$ \mathrm{~F} \$ 7 ; \mathrm{H} 13 ; \mathrm{H} 13-\mathrm{J} 13+\$ \mathrm{~F} \$ 7)$ \\
\hline $\mathrm{L}$ & $=\mathrm{SE}(\mathrm{J} 13>\$ F \$ 7 ; \mathrm{K} 13 ; \mathrm{SE}(\mathrm{H} 13+\mathrm{I} 13>\mathrm{K} 13 ; \mathrm{K} 13 ; \mathrm{H} 13+\mathrm{I} 13))$ \\
\hline $\mathrm{M}$ & $=(-\mathrm{A} 13+1)+\mathrm{SE}(\$ \mathrm{C} \$ 5>\mathrm{H} 13 ; 0 ; \$ C \$ 4)+\mathrm{CONT} . \mathrm{SE}(\$ \mathrm{~L} \$ 13: \mathrm{L} 13 ; "<" \& H 13)$ \\
\hline $\mathrm{N}$ & $\begin{array}{l}=\mathrm{SE}(\$ \mathrm{C} \$ 5>\mathrm{H} 13 ; \mathrm{L} 13-\mathrm{H} 13 ; \mathrm{SE}(\mathrm{E}(\mathrm{H} 13<\$ C \$ 2 ; \mathrm{M} 13<=0) ; \mathrm{MENOR}(\$ \mathrm{~L} \$ 13: \mathrm{L} 13 ; \\
\mathrm{A} 13-\$ \mathrm{C} \$ 4)-\mathrm{H} 13 ; 0))\end{array}$ \\
\hline $\mathrm{O}$ & $\begin{array}{l}=\mathrm{SE}(\mathrm{E}(\mathrm{H} 13<\$ C \$ 2 ; \mathrm{G} 13<>\mathrm{G} 12 ; \mathrm{M} 13<0 ; \mathrm{N} 12>0) ; \mathrm{MENOR}(\$ \mathrm{~L} \$ 13: \mathrm{L} 13 ; \mathrm{A} 13- \\
\$ \mathrm{C} \$ 4-1)-\mathrm{H} 13 ; 0)\end{array}$ \\
\hline
\end{tabular}

Fonte: Elaboração própria

Entre todas as equações apresentadas no Quadro 2, apenas as da coluna B e $C$ exigem modificação antes de serem copiadas para as linhas seguintes. $O$ quarto argumento da função CB.Weibull(<Parâmetros>), \$C\$6 na coluna B e \$C\$7 na coluna $\mathrm{C}$, deve ser removido. Outra observação que deve ser repetida em relação ao modelo da seção 3.1, é que o número de linhas deve garantir que a última demanda por peça na simulação (última linha da coluna $\mathrm{H}$ ) ocorra depois do tempo total de simulação (célula C2).

As Equações 7 e 8 equivalem, respectivamente, às equações das colunas $C$, D e E indicadas no Quadro 2.

$f_{D}(n)=\left\{\begin{array}{cc}P+f_{D}(n-1) & \text { se } T F A_{n}>P \\ T F A_{n}+f_{D}(n-1) & \text { se } T F A_{n} \leq P\end{array}\right.$
$f_{E}(n)= \begin{cases}P+f_{E}(n-1)+\theta & \text { se } T F B_{n}>P \\ T F B_{n}+f_{E}(n-1) & \text { se } T F B_{n} \leq P\end{cases}$

onde $\mathrm{n}$ indica o número do evento de demanda por peça, $\mathrm{P}$ o intervalo de substituição preventiva, $T F A_{n}$ e $T F B_{n}$ o tempo até a falha simulado para a peça $\mathrm{A} \mathrm{e}$ $B$, respectivamente, na linha do evento n. $O$ parâmetro $\theta$ deve assumir um valor muito pequeno que serve para diferenciar qual manutenção preventiva acontece primeiro, caso elas coincidam. Destaca-se que $f_{D}(0)$ e $f_{E}(0)$ são iguais a zero. 
Como a coluna $F$ simplesmente ordena os valores das colunas $D$ e $E$ em ordem crescente, o menor valor deste conjunto é pertinente a $f_{F}(1)$, o segundo menor a $f_{F}(2)$ e assim sucessivamente. As Equações 9, 10, 11, 11, 12, 13, 14, 15 e 16 equivalem, respectivamente, às equações das colunas $G, H, J, K, L, M, N$ e O indicadas no Quadro 2.

$f_{G}(n)= \begin{cases}A & \text { se } f_{F}(n) \in f_{D} \\ B & \text { se } f_{F}(n) \in f_{E}\end{cases}$

$f_{H}(n)= \begin{cases}f_{F}(n)+\sum_{i=1}^{n} \sum_{f_{G}(i)=A} f_{N}(i) & \text { se } f_{G}(n)=A \\ f_{F}(n)+\sum_{i=1}^{n} \sum_{f_{G}(i)=B} f_{N}(i) & \text { se } f_{G}(n)=B\end{cases}$

$f_{J}(n)= \begin{cases}f_{B}\left(\sum_{i=1}^{n} \sum_{f_{G}(i)=A}^{1}\right) & \text { se } f_{G}(n)=A \\ f_{C}\left(\sum_{i=1}^{n} \sum_{f_{G}(i)=B}^{1}\right) & \text { se } f_{G}(n)=B\end{cases}$

$f_{K}(n)=\left\{\begin{array}{cc}f_{H}(n) & \text { se } f_{J}(n)>P \\ f_{H}(n)-f_{J}(n)+P & \text { se } f_{J}(n) \leq P\end{array}\right.$

$f_{L}(n)=\left\{\begin{array}{l}f_{K}(n) \text { se } f_{J}(n)>P \\ f_{L 1}(n) \text { se } f_{J}(n) \leq P\end{array}\right.$

$f_{L 1}(n)=\left\{\begin{array}{cl}f_{K}(n) & \text { se } f_{H}(n)+T L_{n}>f_{K}(n) \\ f_{H}(n)+T L_{n} & \text { se } f_{H}(n)+T L_{n} \leq f_{K}(n)\end{array}\right.$

$f_{M}(n)=(-n+1)+f_{M 1}(n)+\sum_{i=1}^{n} \sum_{f_{L}(i)<f_{H}(n)} 1$

$f_{M 1}(n)= \begin{cases}0 & \text { se } I>f_{H}(n) \\ M & \text { se } I \leq f_{H}(n)\end{cases}$

$f_{N}(n)=\left\{\begin{array}{cc}f_{L}(n)-f_{H}(n) & \text { se } I>f_{H}(n) \\ f_{N 1}(n) & \text { se } I \leq f_{H}(n)\end{array}\right.$

$f_{N 1}(n)=\left\{\begin{array}{cc}f_{L}(n-M)-f_{H}(n) & \text { se } f_{H}(n)<H \& f_{M}(n) \leq 0 \\ 0 & \text { caso contrário }\end{array}\right.$

$f_{O}(n)=\left\{\begin{array}{cc}f_{O 1}(n) & \text { se } f_{H}(n)<H \& f_{G}(n) \neq f_{G}(n-1) \& f_{M}(n)<0 \& f_{N}(n-1)>0 \\ 0 & \text { caso contrário }\end{array}\right.$

$f_{01}(n)=f_{L}(n-M-1)-f_{H}(n)$

onde A e B são definidos como texto, $T L_{n}$ indica o lead time de entrega da peça demandada após o evento n, I indica o momento de início do estoque em questão, M o nível máximo de estoque e $\mathrm{H}$ o horizonte de simulação. $f_{N}(0)$ é igual a zero. 
As duas variáveis de resposta deste modelo são:

- tempo total indisponível devido à espera por peças faltantes (no caso o mancal guia) em estoque (Célula C3). Na planilha da Figura 6 a equação utilizada é a =SOMA(O13:O67), equivalente a expressão:

Tempo indisponível por espera $=\sum_{i=1}^{N} f_{o}(i)$

onde $\mathrm{N}$ representa o número total de eventos simulados.

O modelo da Figura 6 representa um item de classificação "Dois em paralelo". No caso de itens de classificação "Dois em série", a coluna O não deve existir e a célula $\mathrm{C} 3$ deve fazer referência à coluna $\mathrm{N}$;

- valor total gasto simulado com a aquisição de peças (Célula C9). A equação utilizada na planilha representada na Figura 6 é = (CONT.SE(H13:H67;"<"\&C2)+C4)^C8, equivalente à expressão:

Valor gasto com aquisição do item $=\left(M+\sum_{i=1}^{n} \sum_{f_{c}(n) \leq H} 1\right) * C$

onde C representa o custo unitário de aquisição da peça.

As equações das células C3 e C9, além das presentes nas colunas F, G e J, devem se ajustar ao número de linhas utilizadas para a simulação de cada item.

Na Figura 7, destaca-se o histograma da variável tempo indisponível por espera devido à falta de filtro autolimpante (célula C3 da Figura 6) para um horizonte de análise de 10 anos dado um cenário de inexistência de estoques de peças sobressalentes (nível máximo de estoque igual a zero).

Como o filtro autolimpante se encontra dentro de um subsistema redundante, apesar do parâmetro de escala da distribuição Weibull utilizada para modelar o tempo até a falha ser menor do que um ano, mais de $70 \%$ dos valores simulados para a variável da célula foram são iguais a zero. A média simulada foi de 32 horas, percentil 95 de 176 horas e valor máximo de 349 horas. Apesar da redundância, a inexistência de estoque para o item filtro autolimpante ainda gera potencial indisponibilidade ao sistema, porém não tão grande quando a inexistência de estoque de mancal guia que implica em uma média simulada de 221 horas indisponíveis por falta de peça. Quando a simulação foi refeita para considerar nível de estoque máximo de uma peça, nenhum evento de indisponibilidade decorrente da falta do item em estoque foi simulado. 
Figura 7 - Tempo indisponível por espera do subsistema do filtro autolimpante

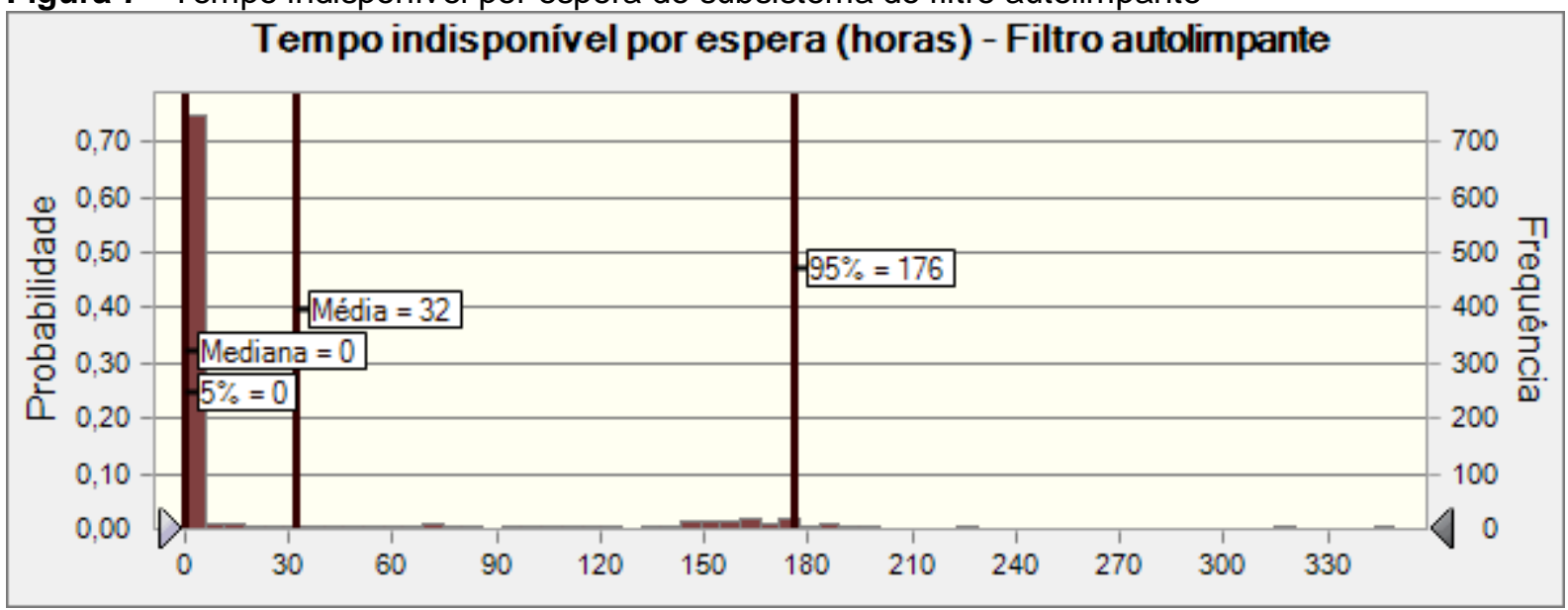

Fonte: Elaboração própria com o auxílio do software Crystal Ball

Na Figura 8 encontra-se, ainda considerando a inexistência de estoque de sobressalentes, o histograma da simulação do valor previsto para aquisição de filtro autolimpante durante o período de análise.

Figura 8 - Valor gasto com aquisição de filtros autolimpante

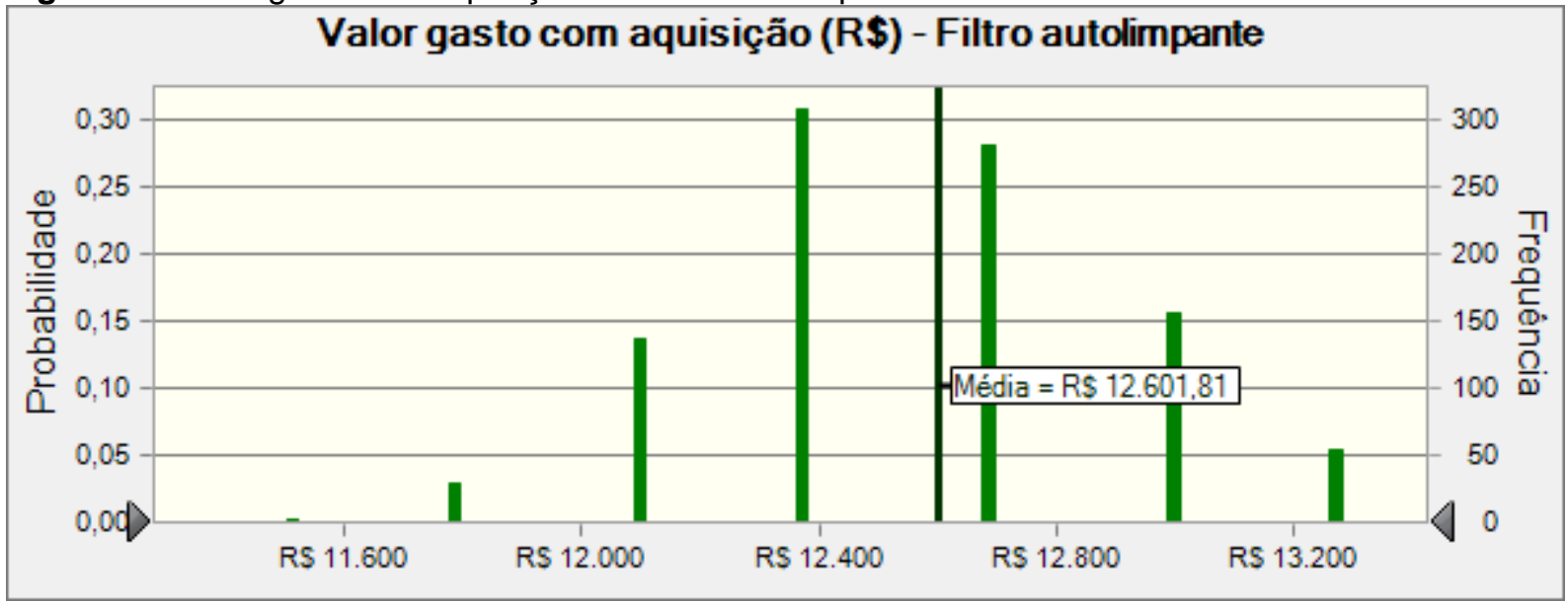

Fonte: Elaboração própria com o auxílio do software Crystal Ball

Na simulação o valor mínimo foi de $\mathrm{R} \$ 11.505$ e o valor máximo de $\mathrm{R} \$$ 13.275, o que equivale a, respectivamente, 39 e 45 unidades adquiridas por $\mathrm{R} \$ 295$ cada. O valor de $\mathrm{R} \$ 12.390$ foi o que mais se repetiu (aproximadamente $30,7 \%$ das simulações) e a média simulada foi de $R$ \$ 12.601,81. Na simulação refeita para nível de estoque máximo de uma peça, a nova média foi de $\mathrm{R} \$ 13.093,90$. Desta maneira, a mudança na política de estoque de zero para uma peça provocou um incremento de 3,9\% no valor médio simulado. 
Os modelos discutidos na seção 3.1 e 3.2 permitiram a análise dos impactos da política de estoque de diferentes tipos de itens de forma isolada. Naturalmente, a estratégia de estoque considerou as restrições de orçamento, em que os níveis de estoque de diferentes itens competiam entre si. Por este motivo, a seguir discute-se o modelo de otimização estocástica que considera o impacto agregado da política de estoque de cada item no sistema como um todo.

\subsection{Modelo de otimização estocástica sistêmica}

Nesta seção, foi desenvolvido um modelo para determinar o nível de estoques de cada um dos itens de um sistema, considerando-se restrições de orçamento. Na Figura 2 (aba "Planilha resumo") constam os parâmetros de entrada utilizados no modelo. Na Figura 9 (também aba "Planilha resumo"), são apresentadas as variáveis de decisão, as restrições e variáveis de resposta do modelo.

A coluna $M$ se refere ao nível de estoque máximo de cada item a ser determinado pelo algorítmico de otimização. As colunas $\mathrm{N}$ e $\mathrm{O}$ indicam, respectivamente, o limite inferior e superior desta variável de decisão discreta.

$\mathrm{Na}$ coluna $\mathrm{P}$ as células são usadas para definir o momento inicial em o estoque de um determinado item passa a existir. Apenas as células com fundo amarelo são definidas como variáveis de decisão no software Crystal Ball. As colunas Q, R e S representam, respectivamente, o limite inferior, superior e passo (incremento) da variável discretizada da coluna $P$.

No modelo apresentado, o intervalo de substituição preventiva de um item foi tratado como uma variável de entrada previamente definida (ver Figura 2). Porém, poderia ter sido tratado como uma variável de decisão a ser otimizada, desde que o número de linhas na planilha de simulação deste item (como explicado nas seções 3.1 e 3.2) garanta que a simulação não acabe antes do final do horizonte de análise.

O propósito da otimização empregada foi o de definir os valores das variáveis de decisão para minimizar a média do tempo total indisponível devido à espera por peças de reposição (célula T28). A região T5:T26 busca o tempo indisponível por falta de estoque de cada item na sua respectiva aba de simulação. Na célula T15, por exemplo, busca o valor referente ao mancal guia com a equação ='Mancal 
guia'!C3. A Célula T28 utiliza a equação =SOMA(T5:T26) para somar todo o tempo indisponível por falta de peça, ou seja, é a função objetivo do problema.

Figura 9 - Variáveis de decisão e de resposta, aba "Planilha resumo"

\begin{tabular}{|c|c|c|c|c|c|c|c|c|c|c|}
\hline 1 & L & $\mathrm{M}$ & $\mathrm{N}$ & 0 & $P$ & Q & $\mathrm{R}$ & $\mathrm{s}$ & $\mathrm{T}$ & u \\
\hline 2 & & \multicolumn{3}{|c|}{$\begin{array}{l}\text { Decisão sobre o nível } \\
\text { máximo de estoque } \\
\text { (em unidades) }\end{array}$} & \multicolumn{4}{|c|}{$\begin{array}{l}\text { Decisão sobre o início do estoque } \\
\text { (horas) }\end{array}$} & \multicolumn{2}{|c|}{ Variáveis de resposta simuladas } \\
\hline 4 & Itens & $\begin{array}{c}\text { Nível } \\
\text { escolhido }\end{array}$ & \begin{tabular}{c|} 
Limite \\
inferior
\end{tabular} & $\begin{array}{c}\text { Limite } \\
\text { superior }\end{array}$ & $\begin{array}{c}\text { Início } \\
\text { escolhido }\end{array}$ & $\begin{array}{l}\text { Limite } \\
\text { inferior }\end{array}$ & $\begin{array}{l}\text { Limite } \\
\text { inferior }\end{array}$ & Passo & $\begin{array}{c}\text { Tempo } \\
\text { indisponivel por } \\
\text { espera (horas) }\end{array}$ & $\begin{array}{c}\text { Valor gasto com } \\
\text { um determinado } \\
\text { item (R\$̦) }\end{array}$ \\
\hline 5 & Anel coletor da excitação & 2 & 0 & 3 & 0 & & & & 0 & $\mathrm{R} \$ 30.600$ \\
\hline 6 & Bucha - TE & 0 & 0 & 1 & 17.520 & 17.520 & 78.840 & 8.760 & 0 & $\mathrm{R} \$ 0$ \\
\hline 7 & Câmera de extinção & 1 & 0 & 1 & 0 & & & & 0 & $\mathrm{R} \$ 24.660$ \\
\hline 8 & Capacitor - CS & 0 & 0 & 1 & 0 & & & & 0 & $\mathrm{R} \$ 0$ \\
\hline 9 & Disjuntor de sincronismo & 0 & 0 & 1 & 0 & & & & 0 & $\mathrm{R} \$ 0$ \\
\hline 10 & Disjuntor principal & 0 & 0 & 1 & 0 & & & & 451 & $\mathrm{R} \$ 17.880$ \\
\hline 11 & Enrolamento - TE & 0 & 0 & 1 & 0 & & & & 0 & $\mathrm{R} \$ 0$ \\
\hline 12 & Filtro autolimpante & 2 & 0 & 6 & 0 & & & & 0 & $\mathrm{R} \$ 12.685$ \\
\hline 13 & Filtro cesto & 3 & 0 & 6 & 0 & & & & 0 & $R \$ 5.175$ \\
\hline 14 & Isolador & 0 & 0 & 1 & 0 & & & & 0 & $\mathrm{R} \$ 0$ \\
\hline 15 & Mancal guia & 2 & 0 & 3 & 0 & & & & 0 & $\mathrm{R} \$ 28.500$ \\
\hline 16 & Núcleo - TE & 0 & 0 & 1 & 26.280 & 26.280 & 78.840 & 8.760 & 1.404 & $\mathrm{R} \$ 102.100$ \\
\hline 17 & Para-raios & 1 & 0 & 3 & 0 & & & & 0 & $\mathrm{R} \$ 80.720$ \\
\hline 18 & Relé de bloqueio & 1 & 0 & 6 & 0 & & & & 0 & $R \$ 6.500$ \\
\hline 19 & Relé de gás - TE & 3 & 0 & 3 & 0 & & & & 0 & $R \$ 6.300$ \\
\hline 20 & Relé de proteção & 1 & 0 & 6 & 0 & & & & 0 & $\mathrm{R} \$ 4.800$ \\
\hline 21 & Resistor de aterramento & 1 & 0 & 2 & 0 & & & & 0 & $\mathrm{R} \$ 26.200$ \\
\hline 22 & Sapata & 2 & 0 & 4 & 0 & & & & 0 & $\mathrm{R} \$ 61.320$ \\
\hline 23 & Sensor de pressão & 0 & 0 & 1 & 8.760 & 8.760 & 78.840 & 8.760 & 0 & $\mathrm{R} \$ 0$ \\
\hline 24 & Transformador de corrente & 0 & 0 & 1 & 17.520 & 8.760 & 78.840 & 8.760 & 0 & $\mathrm{R} \$ 0$ \\
\hline 25 & Transformador de potencial & 1 & 0 & 1 & 8.760 & 8.760 & 78.840 & 8.760 & 0 & $\mathrm{R} \$ 21.200$ \\
\hline 26 & Vedação de eixo da turbina & 1 & 0 & 1 & 17.520 & 17.520 & 78.840 & 8.760 & 0 & $\mathrm{R} \$ 5.910$ \\
\hline 27 & & & & & & & & & $\begin{array}{l}\text { Somatória do } \\
\text { tempo } \\
\text { indisponivel } \\
\text { (horas) }\end{array}$ & $\begin{array}{l}\text { Somatória do } \\
\text { valor gasto com } \\
\text { estoque (R\$) }\end{array}$ \\
\hline 28 & & & & & & & & & 1.855 & $\mathrm{R} \$ 434.550$ \\
\hline 14 & A $\mapsto \mid<$ Transformador de ce & orrente & Transform & mador de $p$ & & Vedação d & lis e eis & & Planilha resumo & IIII \\
\hline
\end{tabular}

Fonte: Elaboração própria

O vetor composto pelas células U5:U26 tem a função de registrar o valor do total gasto com um determinado item composto por (a) peças previamente existentes no estoque e (b) demanda por peças simulada. A célula U15, por exemplo, utiliza a equação ='Mancal guia'!C8. A célula U28 utiliza a equação =SOMA(U5:U26) para somar o custo simulado de cada um dos itens considerados. Esta variável de resposta é utilizada para a definição da restrição estatística de custo na otimização. A política de estoque é considerada infactível se exceder o custo máximo pré-determinado em mais de $5 \%$ das simulações.

O computador utilizado nas simulações e otimizações foi um Toshiba modelo Satellite U845W com processador Intel (R) Core (TM) I5-3317U CPU G 1.70 GHz 
com 6 GB de memória RAM, sistema operacional Windows 7 de 64-bit e Microsoft Excel 2010. Cada otimização teve duração de 3 horas. Como destacado na célula B2 da aba "Planilha resumo" o horizonte de simulação definido foi de 87.600 horas (ou 10 anos de operação).

Baseado na restrição de que o orçamento de $\mathrm{R} \$ 400.000$ não pode ser excedido em mais que 5\% das simulações, na Figura 10 mostra-se o histograma simulado da somatória do tempo indisponível devido à espera por peças dado o nível de estoque otimizado.

Figura 10 - Tempo indisponível devido à espera por peças no caso em que o orçamento possui restrição de percentil 95 menor ou igual a $\mathrm{R} \$ 400.000$

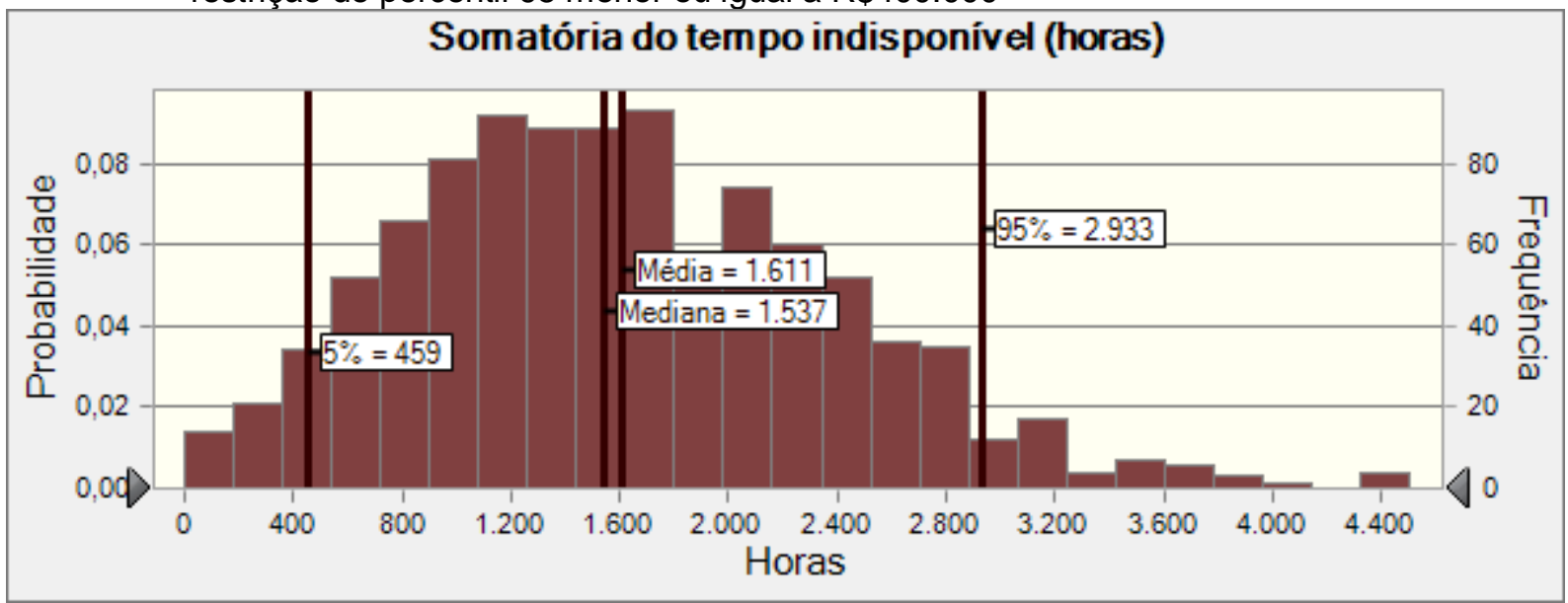

Fonte: Elaboração própria com o auxílio do software Crystal Ball

Os valores simulados variam de um mínimo de zero hora até um máximo de 4.501 horas, com média de 1.611 horas, mediana de 1.537 horas e desvio padrão de 779 horas. O valor mínimo simulado de zero hora só se repete em apenas 0,2\% das simulações.

A observância dos percentis encontrados apresenta grande importância no estudo da dispersão dos dados. Os percentis 5 e 95 são iguais a, respectivamente, 459 e 2.933 horas. Estes valores indicam que, de acordo com o modelo de simulação, existe uma certeza de $90 \%$ de que o valor do tempo total indisponível esteja entre 459 e 2.933 horas.

Observa-se que a simulação permite ao analista entender como cada política de estoque de peças de reposição (composta pelos níveis de estoque de cada item) afeta as estatísticas do tempo total indisponível por falta de peças. Assim, esta abordagem permitiu então comparar o desempenho de diferentes estratégias. Para 
ilustrar esta comparação, na Figura 11 mostra-se o resultado de simulação na forma de histograma da variável tem total indisponível devido à espera por peças para o nível de estoque otimizado considerando que o custo total com aquisição de peças não ultrapasse R\$ 550.000 em mais de $5 \%$ das simulações.

Figura 11 - Tempo indisponível devido à espera por peças no caso em orçamento possui restrição de percentil 95 menor ou igual a $\mathrm{R} \$ 550.000$

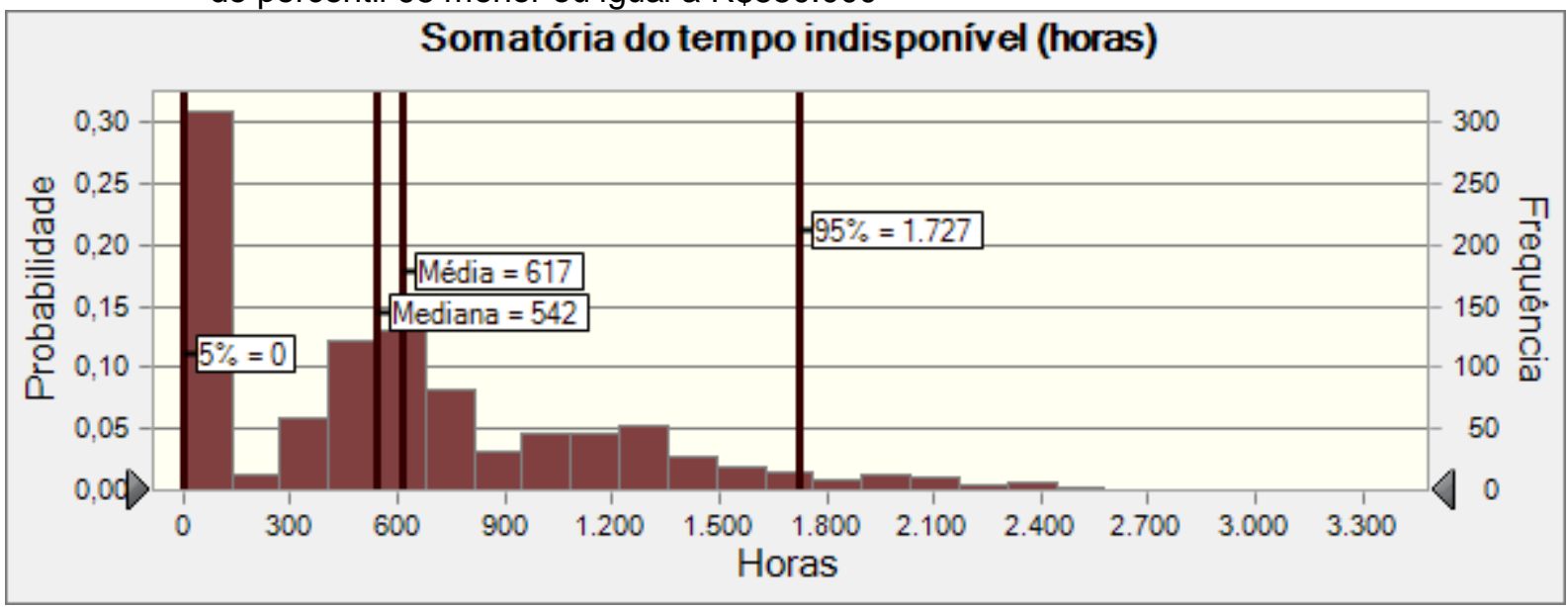

Fonte: Elaboração própria com o auxílio do software Crystal Ball

O valor de zero hora indisponível por falta de peça se repete em mais de $30 \%$ das simulações. Em outras palavras, é possível, porém não provável, que a política simulada não afete negativamente a disponibilidade do sistema por falta de peças. Como indica a mediana, metade dos valores simulados encontra-se abaixo de aproximadamente 542 horas.

A simulação pertinente a Figura 11 gerou média de 617 horas e o percentil 95 de 1.727 horas. Enquanto que a simulação pertinente a Figura 10 gerou média de 1.611 horas e o percentil 95 de 2.933 horas. Baseado neste tipo de comparação, o gestor de estoque pode entender como o desempenho de um sistema é afetado pela política de estoque otimizada para diferentes limites de orçamento.

$\mathrm{Na}$ Tabela 1, encontram-se as estatísticas geradas pela simulação do tempo total indisponível do sistema devido à espera por peças de acordo com a política de estoque otimizado para diferentes níveis de orçamento.

Se a restrição de custo de $\mathrm{R} \$ 550.000$ for aumentada para $\mathrm{R} \$ 600.000$ (aumento de 9,1\%), o tempo médio indisponível é reduzida em 55,9\% (de 617 para 272 horas). 
Tabela 1 - Estatísticas de cada solução otimizada respeitando sua respectiva restrição estatística

\begin{tabular}{|c|c|c|c|c|c|}
\hline \multicolumn{2}{|c|}{ Somatória do valor gasto com estoque } & \multicolumn{4}{|c|}{ Somatória do tempo indisponível por espera } \\
\hline $\begin{array}{c}\text { Valor mínimo aceitável } \\
\text { para o percentil 95 (R\$) }\end{array}$ & Média (R\$) & $\begin{array}{c}\text { Média } \\
\text { (horas) }\end{array}$ & $\begin{array}{c}\text { Percentil } \\
5 \text { (horas) }\end{array}$ & $\begin{array}{c}\text { Percentil } \\
50 \text { (horas) }\end{array}$ & $\begin{array}{c}\text { Percentil } \\
95 \text { (horas) }\end{array}$ \\
\hline 350.000 & 262.292 & 3.411 & 1.866 & 3.384 & 5.147 \\
400.000 & 313.823 & 1.611 & 459 & 1.537 & 2.933 \\
450.000 & 355.554 & 1.420 & 343 & 1.315 & 2.805 \\
500.000 & 409.621 & 684 & 0 & 628 & 1.747 \\
550.000 & 420.129 & 617 & 0 & 542 & 1.727 \\
600.000 & 506.538 & 272 & 0 & 0 & 986 \\
650.000 & 527.337 & 164 & 0 & 0 & 776 \\
700.000 & 580.328 & 7 & 0 & 0 & 0 \\
\hline
\end{tabular}

Fonte: Elaboração própria

Entender a relação entre as restrições sobre o custo (primeira coluna da Tabela 1) e as estatísticas da variável de resposta do modelo (quatro últimas colunas) considerando as soluções otimizadas para cada restrição de custo é muito importante para a tomada de decisões gerenciais. A estratégia de manutenção deve estipular os níveis de estoque de modo a utilizar os recursos finitos de maneira eficiente e atender às metas operacionais do sistema. Se, por exemplo, for intolerável que o tempo de indisponível por espera de peças durante 10 anos seja maior ou igual a 2.200 horas com $5 \%$ de probabilidade, então o orçamento de até $R$ \$ 450.000 com 95\% de certeza é insuficiente.

Um resumo dos dados da Tabela 1 pode ser observado no gráfico da Figura 12.

Nota-se que a restrição estatística sobre o orçamento não apresenta relação linear com as estatísticas da função objetivo. Com o gráfico apresentado, a gerência pode ter o real conhecimento de como o planejamento do orçamento com peças de reposição pode afetar o desempenho do sistema.

A restrição estatística sobre o valor total gasto com peças ao longo de 10 anos poderia ser feita também em relação a cada ano isoladamente. Nesta situação, a variável sobre o momento de início do estoque (região P5:P26 da aba "Planilha resumo") ganha importância por permitir que o estoque de uma determinada peça só passe a existir a partir de determinado momento futuro. 
Figura 12 - Gráfico com o resumo das estatísticas simuladas para cada nível de restrição de custo

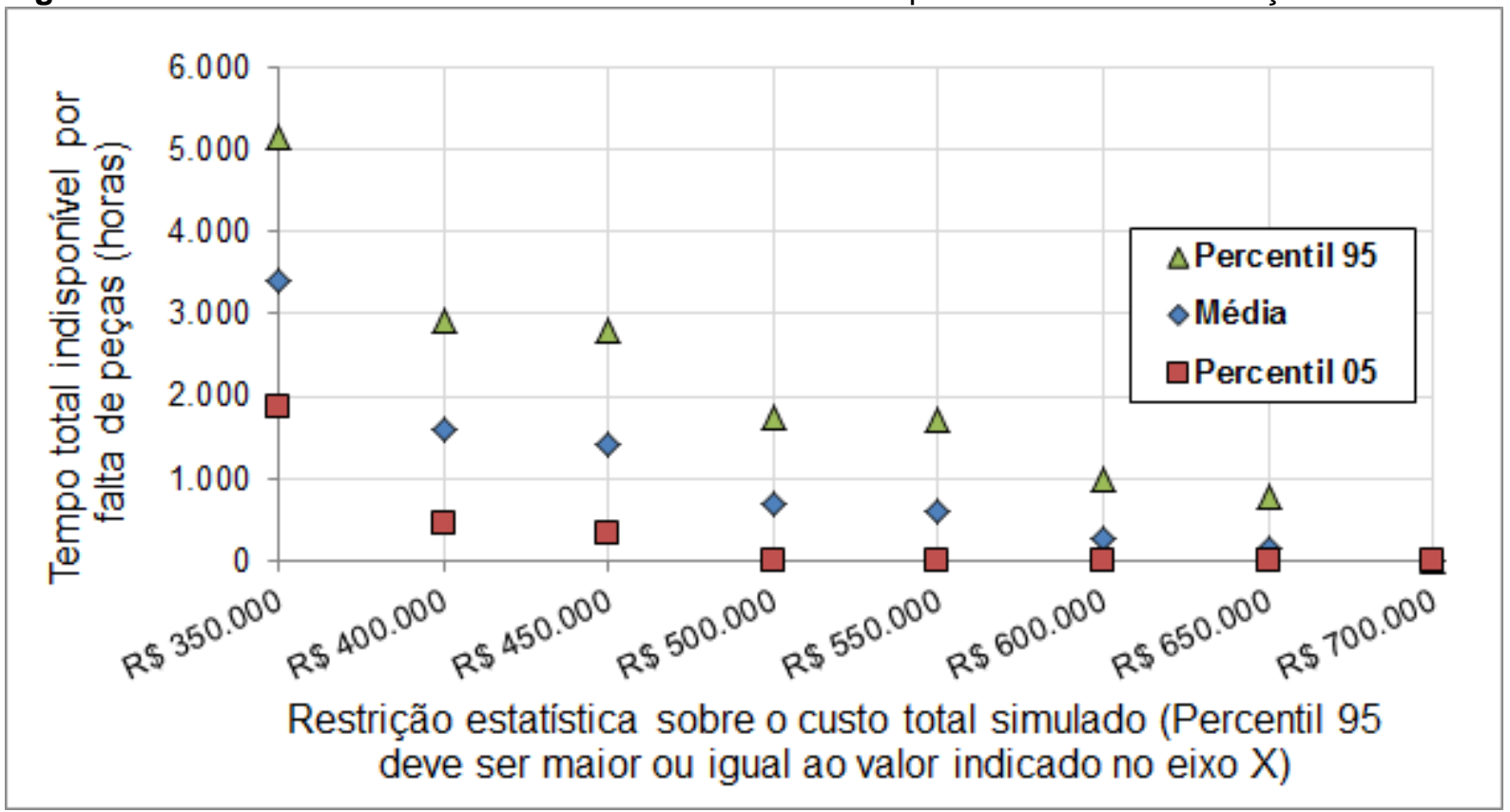

Fonte: Elaboração própria

\section{CONSIDERAÇÕES FINAIS}

As principais conclusões decorrentes do processo de desenvolvimento do modelo, simulação e otimização são:

- os modelos baseados em simulação de Monte Carlo permitiram representar as particularidades de um sistema que passa por manutenção de maneira mais próxima à realidade, se comparado a modelos analíticos. Isto se justifica principalmente pela capacidade de considerar diferentes tipos de distribuição de probabilidade e por permitir o uso de lógicas mais complexas;

- a modelagem em planilhas, apesar de pouco utilizada em meios acadêmicos, permitiu que modelos fossem mais facilmente recebidos por profissionais da indústria. Além disso, add-ins para Excel como o Crystal Ball, @Risk e ModelRisk, são acessíveis inclusive para pequenas empresas, o que aumenta o potencial de uso do modelo;

- o uso do modelo de simulação desenvolvido, quando aplicado a otimização estocástica, permite a profissionais encontrar boas soluções para a política de estoque de sobressalentes acordo com diferentes valores de restrição de orçamento durante um horizonte de tempo finito. 
- a abordagem apresentada é capaz de revelar a relação entre as estatísticas do tempo total indisponível por falta de peças e as restrições de orçamento (como na Figura 12), permitindo que aos gestores tenham uma clara percepção sobre o trade-off entre indisponibilidade e custo;

- os autores observaram a necessidade de trabalhos futuros que incluam na modelagem a existência de estoque intermediário de produto em processo para amortecer a falta de peça em determinada parte de um sistema, a qual é muito relevante para os setores de mineração e siderurgia.

\section{AGRADECIMENTOS}

Os autores agradecem à ANEEL e AES-Tietê pelo financiamento para o desenvolvimento deste trabalho dentro do projeto de P\&D.

\section{REFERÊNCIAS}

ALRABGHI, Abdullah; TIWARI, Ashutosh; ALABDULKARIM, Abdullah. Simulation based optimization of joint maintenance and inventory for multi-components manufacturing systems. In: Proceedings of the 2013 Winter Simulation Conference: Simulation: Making Decisions in a Complex World. IEEE Press, 2013. p. 1109-1119. https://doi.org/10.1109/WSC.2013.6721500

ALRABGHI, Abdullah; TIWARI, Ashutosh. State of the art in simulation-based optimisation for maintenance systems. Computers \& Industrial Engineering, v. 82, p. 167-182, 2015. https://doi.org/10.1016/j.cie.2014.12.022

ARMENZONI, Mattia; MONTANARI, R.; VIGNALI, G.; BOTTANI, E.; FERRETTI, G.; SOLARI, F.; RINALDI, M. An integrated approach for demand forecasting and inventory management optimisation of spare parts. International Journal of Simulation and Process Modelling, v. 10, n. 3, p. 233-240, 2015. http://dx.doi.org/10.1504/IJSPM.2015.071375

BASTEN, R. J. I.; VAN HOUTUM, G. J. System-oriented inventory models for spare parts. Surveys in operations research and management science, v. 19, n. 1, p. 34-55, 2014. https://doi.org/10.1016/j.sorms.2014.05.002

BIROLINI, Alessandro. Reliability engineering: theory and practice 7. ed. Springer-Verlag Berlin Heidelberg, 2014. http://dx.doi.org/10.1007/978-3-642-39535-2

BJARNASON, Erik Tryggvi Striz; TAGHIPOUR, S. Optimizing simultaneously inspection interval and inventory levels (s, S) for a k-out-of-n system. In: Reliability and Maintainability Symposium (RAMS), 2014 Annual. IEEE, 2014. p. 1-6. http://dx.doi.org/10.1109/RAMS.2014.6798463

DOLGUI, Alexandre; PASHKEVICH, Maksim. Demand forecasting for multiple slow-moving items with short requests history and unequal demand variance. International Journal of Production Economics, v. 112, n. 2, p. 885-894, 2008.

http://dx.doi.org/10.1016/j.ijpe.2007.07.008 
DOS SANTOS, Nilis Adriano; SELLITTO, Miguel Afonso. Estratégia de manutenção e aumento da disponibilidade de um posto de compressão de gases na indústria petrolífera. Revista Produção Online, v. 16, n. 1, p. 77-103, 2016.

http://dx.doi.org/10.14488/1676-1901.v16i1.1905

EBELING, Charles E. An introduction to reliability and maintainability engineering $\mathbf{2 e}$. Waveland Press, 2010.

FARASYN, Ingrid; PERKOZ, Koray; VAN DE VELDE, Wim. Spreadsheet models for inventory target setting at Procter \& Gamble. Interfaces, v. 38, n. 4, p. 241-250, 2008. http://dx.doi.org/10.1287/inte.1080.0345

GAN, Shuyuan.; ZHANG, Z.; ZHOU, Y.; SHI, J. Joint optimization of maintenance, buffer, and spare parts for a production system. Applied Mathematical Modelling. v.39, n.19, p.6032-6042, 2015. http://dx.doi.org/10.1016/j.apm.2015.01.035

GE, Fujian; WEN, Meilin; HAN, Qiao. Multi-objective spare parts inventory optimization in uncertain environment. In: Prognostics and System Health Management Conference (PHM-Chengdu), 2016. IEEE, 2016. p. 1-5. https://doi.org/10.1109/PHM.2016.7819833

HAGMARK, P. E.; PERNU, H. Risk evaluation of a spare part stock by stochastic simulation. In: ESREL 2006 Conference, Safety and Reliability for Managing Risk. 2006. p. 18-22. http://dx.doi.org/10.13140/2.1.2636.6248

HUISKONEN, Janne. Maintenance spare parts logistics: Special characteristics and strategic choices. International journal of production economics, v. 71, n. 1, p. 125-133, 2001. http://dx.doi.org/10.1016/S0925-5273(00)00112-2

KECECIOGLU, Dimitri. Reliability and life testing handbook. DEStech Publications, Inc, 2002.

KEIZER, Minou CA Olde; TEUNTER, Ruud H.; VELDMAN, Jasper. Joint condition-based maintenance and inventory optimization for systems with multiple components. European Journal of Operational Research, v. 257, n. 1, p. 209-222, 2017.

https://doi.org/10.1016/j.ejor.2016.07.047

LENGU, D.; SYNTETOS, Aris A.; BABAI, M. Zied. Spare parts management: Linking distributional assumptions to demand classification. European Journal of Operational Research, v. 235, n. 3, p. 624-635, 2014. http://dx.doi.org/10.1016/j.ejor.2013.12.043

LYNCH, P.; ADENDORFF, K.; YADAVALLI, V. S. S.; ADETUNJI, O. Optimal spares and preventive maintenance frequencies for constrained industrial systems. Computers \& Industrial Engineering, v. 65, n. 3, p. 378-387, 2013.

http://dx.doi.org/10.1016/j.cie.2013.03.005

NAKAGAWA, Toshio. Stochastic processes: With applications to reliability theory. Springer-Verlag London, 2011. http://dx.doi.org/10.1007/978-0-85729-274-2

NELSON, Wayne B. Applied life data analysis. John Wiley \& Sons, 2005.

POWELL, Stephen. G; BAKER, Keneeth. R. Management Science: The art of modeling with spreadsheets 3d. John Wiley \& Sons, 2009. 
RESENDE, Mauricio GC et al. Scatter search and path-relinking: Fundamentals, advances, and applications. In: Handbook of metaheuristics. Springer US, 2010. p. 87-107. http://dx.doi.org/10.1007/978-1-4419-1665-5 4

SAMAL, Nishith Kumar; PRATIHAR, Dilip Kumar. Joint optimization of preventive maintenance and spare parts inventory using genetic algorithms and particle swarm optimization algorithm. International Journal of System Assurance Engineering and Management, v. 6, n. 3, p. 248-258, 2015. http://dx.doi.org/10.1007/s13198-015-0349-3

SARMAH, S. P.; MOHARANA, U. C. Multi-criteria classification of spare parts inventories-a web based approach. Journal of Quality in Maintenance Engineering, v. 21, n. 4, p. 456477, 2015. http://dx.doi.org/10.1108/JQME-04-2012-0017

SILVA FILHO, Oscar Salviano; CEZARINO, Wagner; RATTO, João. Planejamento agregado da produção: modelagem e solução via planilha Excel \& Solver. Revista Produção Online, v. 9, n. 3, 2009. http://dx.doi.org/10.14488/1676-1901.v9i3.173

SLEPTCHENKO, Andrei; VAN DER HEIJDEN, Matthieu. Joint optimization of redundancy level and spare part inventories. Reliability Engineering \& System Safety, v. 153, p. 64-74, 2016. http://dx.doi.org/10.1016/j.ress.2016.04.006

SMITH, David J. Reliability, Maintainability and Risk 8e: Practical Methods for Engineers Including Reliability Centred Maintenance and Safety-Related Systems. Elsevier, 2011.

TRUSEVYCH, Stephan A.; KWON, Roy H.; JARDINE, Andrew KS. Optimizing Critical Spare Parts and Location Based on the Conditional Value-At-Risk Criterion. The Engineering Economist, v. 59, n. 2, p. 116-135, 2014. http://dx.doi.org/10.1080/0013791X.2013.876795

ZHU, Jianxin; YUAN, W.; XU, P.; LU, Y., CHEN, X. Introduction of the Risk Based Optimization and Risk Criteria Analysis of Spare Inventory in Petrochemical Plant. In: Engineering Asset Management-Systems, Professional Practices and Certification. Springer International Publishing, 2015. p. 1571-1579. http://dx.doi.org/10.2991/978-94$\underline{6239-180-2 \quad 34}$

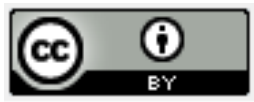

Artigo recebido em 05/08/2016 e aceito para publicação em 27/07/2017

DOI: http://dx.doi.org/10.14488/1676-1901.v17i3.2549 